\title{
MHD simulations of jet acceleration from Keplerian accretion disks
} The effects of disk resistivity

\author{
C. Zanni ${ }^{1,2}$, A. Ferrari ${ }^{2,3}$, R. Rosner ${ }^{3}$, G. Bodo ${ }^{4}$, and S. Massaglia ${ }^{2}$ \\ 1 Laboratoire d'Astrophysique de Grenoble, 414 rue de la Piscine, BP 53, 38041 Grenoble, France \\ e-mail: Claudio.Zanni@obs.ujf-grenoble.fr \\ 2 Dipartimento di Fisica Generale dell'Università, via Pietro Giuria 1, 10125 Torino, Italy \\ e-mail: ferrari@ph.unito.it \\ 3 Department of Astronomy and Astrophysics, University of Chicago, 5640 S. Ellis Av., Chicago, IL 60637, USA \\ e-mail: r-rosner@uchicago.edu \\ 4 Osservatorio Astronomico di Torino, Viale Osservatorio 20, 10025 Pino Torinese, Italy \\ e-mail: bodo@to.astro.it
}

Received 14 September 2006 / Accepted 24 February 2007

\begin{abstract}
Context. Accretion disks and astrophysical jets are used to model many active astrophysical objects, such as young stars, relativistic stars, and active galactic nuclei. However, existing proposals for how these structures may transfer angular momentum and energy from disks to jets through viscous or magnetic torques do not yet provide a full understanding of the physical mechanisms involved. Thus, global stationary solutions have not explained the stability of these structures; and global numerical simulations that include both the disk and jet physics have so far been limited to relatively short time scales and narrow (and possibly astrophysically unlikely) ranges of viscosity and resistivity parameters that may be crucial to defining the coupling of the inflow-outflow dynamics.

Aims. We present self-consistent, time-dependent simulations of supersonic jets launched from magnetized accretion disks, using high-resolution numerical techniques. In particular we study the effects of the disk's magnetic resistivity, parametrized through an $\alpha$-prescription, in determining the properties of the inflow-outflow system. Moreover we analyze under which conditions steady state solutions of the type proposed in the self-similar models of Blandford \& Payne can be reached and maintained in a self-consistent nonlinear stage.

Methods. We used the resistive MHD FLASH code with adaptive mesh refinement (AMR), allowing us to follow the evolution of the structure on a long enough time scale to reach steady state. A detailed analysis of the initial configuration state is given.

Results. We obtain the expected solutions within the axisymmetric $(2.5 \mathrm{D})$ limit. Assuming a magnetic field around equipartition with the thermal pressure of the disk, we show how the characteristics of the disk-jet system, such as the ejection efficiency and the energetics, are affected by the anomalous resistivity acting inside the disk.
\end{abstract}

Key words. accretion, accretion disks - ISM: jets and outflows - galaxies: jets - magnetohydrodynamics (MHD) methods: numerical

\section{Introduction}

Astrophysical jets are an important component in many active astrophysical objects from young stellar objects (YSO) to relativistic stars and galactic nuclei. In particular, Herbig-Haro $(\mathrm{HH})$ outflows are detected in stellar forming regions around $\mathrm{T}$ Tauri stars, characterized mainly by optical emission line spectra (e.g. Reipurth \& Bally 1996). On a much larger scale, relativistic radio jets are accelerated in the innermost cores of active galactic nuclei (AGN) (e.g. Giovannini 2004): their emitting component is synchrotron relativistic electrons, with a cold proton component or, most likely, a Poynting flux electromagnetic component (De Young 2006).

Despite being characterized by extremely different space, time, and energy scales, it is commonly accepted that all these systems derive their energy from accretion onto a central object (Livio 1999), and the physical origin of these supersonic outflows has been related to the dynamical evolution of magnetized accretion disks around a deep gravitational well. Even if the acceleration and collimation mechanisms of jets are still not clear, some basic models have been proposed and shown to be successful (for fairly recent reviews see Pudritz et al. 2006a for YSO and Ferrari 1998, 2004 for AGN). The overall idea is to extract energy and angular momentum from the accreting matter and to feed it into a plasma that is accelerated in two opposite directions along the rotation axis of the disk. Lovelace (1976) and Blandford (1976) independently proposed that this can be done by electromagnetic forces. In particular Blandford \& Payne (1982) derived a steady state MHD solution for an axisymmetric, magnetocentrifugally driven outflow from a Keplerian disk; for the outflow to be launched, the poloidal magnetic field lines must be inclined less than $60^{\circ}$ with respect to the plane of the accretion disk. On the other hand, Sauty \& Tsinganos (1994), Sauty et al. $(2002,2004)$ have derived meridionally self-similar models to study the launching mechanism from the hot corona of the central object.

The magnetocentrifugal mechanism has been the subject of a series of numerical studies (Ustyugova et al. 1999; Ouyed \& Pudritz 1997; Krasnopolsky et al. 1999; Anderson et al. 2004; Fendt 2006; Pudritz 2006b) based on ideal MHD simulations in which the disk is treated as a boundary condition. On one hand, they show how a steady solution can be obtained on a few 
dynamical time scales and how the acceleration, collimation, and stationarity of the outflow depend on the mass loading from the disk and on the magnetic field structure. On the other hand, the back reaction of the outflow on the disk cannot be taken into account.

When the structure of the magnetized accretion disk is included self-consistently in the models, a diffusive mechanism must be introduced inside the disk to balance the shearing due to differential rotation and the inward advection of field lines. Moreover, viscous torques, which can transport angular momentum radially inside the disk itself, also should be taken into account. For instance, Königl (1989), Wardle \& Königl (1993), and Li (1996) have studied the structure of an ambipolar diffusiondominated disk, connecting it with a Blandford \& Payne solution on the disk surface. Ogilvie \& Livio (2001) solved the vertical structure of a thin (optically thick) magnetized disk, taking into account an effective $\alpha$ turbulent viscosity and resistivity.

In a series of papers including turbulent $\alpha$ resistivity (Ferreira \& Pelletier 1995; Ferreira 1997), viscosity (Casse $\&$ Ferreira 2000a), and entropy generation (Casse \& Ferreira 2000 b), the authors calculate radially self-similar stationary solutions of accretion-ejection structures. Two important features emerge clearly from these models. First of all it was shown that, in order to balance the magnetic and gravitational compression on the disk itself, the thermal energy must be around equipartition with the magnetic energy inside the disk. With these conditions, the vertical thermal pressure gradient is the only force that can push the mass on the surface of the disk to be accelerated in the outflow. Second, the magnetic torque must change sign at the disk surface, in order to extract angular momentum from the disk and transfer it to the outflow. This condition determines a strong constraint on the resistive configuration: the magnetic diffusivity must be rather high $(\alpha \sim 1)$ and anisotropic, the diffusion of the poloidal field being smaller than the diffusion of the toroidal component.

The first time-dependent numerical simulations in which the structure of the magnetized accretion disks is included (Uchida $\&$ Shibata 1985 , or more recently Kato et al. 2002) showed that the interaction between a geometrically-thin rotating disk and a large-scale magnetic field that was initially uniform and vertical creates a transient state in which a strong toroidal field is generated that expels matter in the direction perpendicular to the disk plane ("sweeping magnetic twist mechanism"). One of the limits of these models is that the short simulated time scales and the ideal MHD approximation lead to the formation of a transient and highly unstable outflow. Moreover, the low density contrast between the disk and the surrounding corona assumed in Uchida \& Shibata (1985) enables the disk to lose its angular momentum on a very short time scale. These works have been recently updated in Kuwabara et al. (2005) who studied the evolution of a thick magnetized torus assuming a constant resistivity throughout the computational domain. Even if the presence of such a high and uniform resistivity must be justified, the authors show a quasi-stationary outflow launched from the inner radii of the torus.

Up to the present, the best effort to produce an accretionejection structure recurring in time-dependent simulations has been performed by Casse \& Keppens (2002, 2004), who showed how a quasi-stationary jet can be launched from the equipartition regions of a resistive accretion disk. On the other hand, their resistive configuration, isotropic with $\alpha=0.1$, is rather different from the one predicted by the self-similar steady models (Casse \& Ferreira 2000a). Despite the use of a stretched grid, the resolution of these simulations is rather low, and it is likely that numerical dissipative effects are very important.

In the present paper we present a numerical study of resistive MHD axisymmetric accretion-ejection structures, performed with the high resolution code FLASH using adaptive mesh refinement. The aim of the paper is to simulate the disk-jet configuration over long time scales to determine the effects of different configurations of an $\alpha$ resistivity, by varying its value and its degree of anisotropy, in determining the properties of the system. Moreover, we want to test whether a stationary state corresponding to the Blandford \& Payne self-similar solution can be reached and maintained. A critical point in the simulation is of course the choice of the initial configuration, in particular the magnetic configuration and the equilibrium of the disk. We solved an analytic self-similar equilibrium configuration of the disk including gravitational, centrifugal, thermal pressure, and Lorentz forces, a solution that also defines the initial magnetic field. We do not include physical viscosity: as has been shown both in stationary (Casse \& Ferreira 2000a) and time-dependent contexts (Meliani et al. 2006) the viscous torque is less efficient, for conventional values of the disk turbulence $(\alpha \sim 0.1-1$, Prandtl number $\sim 1$ ), than the magnetic in extracting angular momentum from the disk.

This paper is organized as follows. Section 2 is dedicated to illustrating the equations and the numerical code used, and the initial configuration and the boundary conditions are discussed in detail. In Sect. 3 the numerical simulations are presented for different magnetic diffusivities and anisotropies. These parameters are linked to the mass accretion rate, required torque, and liberated accretion energy. The acceleration mechanisms at the disk boundary and far above the disk are discussed, also referring to the established current circuits. The jet ejection efficiency is discussed in Sect. 4 in relation to the accretion rates. In Sect. 5 we discuss the angular momentum transport, and in Sect. 6 the energy budget of the inflow/outflow system is evaluated. In Sect. 7 we test whether one of our cases can be characterized as a stationary solution. Comments about the effect of Ohmic dissipation in the disk are given in Sect. 8. Finally, Sect. 9 summarizes our results and puts them in the context of other analytical and numerical models.

\section{The numerical model}

\subsection{MHD equations}

We model the interaction between an accretion disk and the magnetic field that threads it within a resistive MHD framework. The system of equations that we solve numerically therefore conveys the conservation of mass:

$\frac{\partial \rho}{\partial t}+\nabla \cdot(\rho \boldsymbol{u})=0$

where $\rho$ is the mass density and $\boldsymbol{u}$ the flow speed. The momentum equation is

$\frac{\partial \rho \boldsymbol{u}}{\partial t}+\nabla \cdot\left[\rho \boldsymbol{u} \boldsymbol{u}+\left(P+\frac{\boldsymbol{B} \cdot \boldsymbol{B}}{2}\right) \boldsymbol{I}-\boldsymbol{B} \boldsymbol{B}\right]+\rho \nabla \Phi_{\mathrm{g}}=0$

where $P$ is the thermal pressure and $\boldsymbol{B}$ is the magnetic field. This equation takes into account the action of thermal pressure gradients, Lorentz forces, and gravity as determined by the potential $\Phi_{\mathrm{g}}=-G M / \sqrt{r^{2}+z^{2}}$ representative of the gravitational field of a central object of mass $M$. The evolution of the magnetic field is determined by the induction equation (Faraday's law):

$\frac{\partial \boldsymbol{B}}{\partial t}+\nabla \times \boldsymbol{E}=0$ 
where the electric field $\boldsymbol{E}$ is determined by the Ohm's law $\boldsymbol{E}=-\boldsymbol{u} \times \boldsymbol{B}+\overline{\overline{\boldsymbol{\eta}}} \boldsymbol{J}$. Notice that the equations are written in a non-dimensional form, hence without $4 \pi$ and $\mu_{0}$ coefficients. The electric current $\boldsymbol{J}$ appearing in the Ohm's relation is determined by Ampere's law $\boldsymbol{J}=\nabla \times \boldsymbol{B}$. The magnetic resistivity $\overline{\overline{\boldsymbol{\eta}}}$ is indicated as a two-tensor to take anisotropic diffusive effects into account. In our simulations we will consider a diagonal resistivity tensor $\eta_{i j}$ whose non-zero components are $\eta_{\phi \phi}=\eta_{\mathrm{m}}$ and $\eta_{r r}=\eta_{z z}=\eta_{\mathrm{m}}^{\prime}$.

Finally the conservation of energy is expressed by

$\frac{\partial e}{\partial t}+\nabla \cdot\left[\left(e+P+\frac{\boldsymbol{B} \cdot \boldsymbol{B}}{2}\right) \boldsymbol{u}-(\boldsymbol{u} \cdot \boldsymbol{B}) \boldsymbol{B}+\overline{\overline{\boldsymbol{\eta}}} \boldsymbol{J} \times \boldsymbol{B}\right]=-\Lambda_{\mathrm{cool}}$,

where the total energy density

$e=\frac{P}{\gamma-1}+\frac{\rho \boldsymbol{u} \cdot \boldsymbol{u}}{2}+\frac{\boldsymbol{B} \cdot \boldsymbol{B}}{2}+\rho \Phi_{\mathrm{g}}$

is given by the sum of thermal, kinetic, magnetic and gravitational energy. Here, $\gamma=5 / 3$ is the polytropic index of the gas, and $\Lambda_{\text {cool }}$ is a cooling term defined by the parameter $0<f<1$ :

$f=\frac{\Lambda_{\text {cool }}}{\Lambda_{\text {diss }}}$

given by the ratio between the specific radiated energy and the Ohmic heating term $\Lambda_{\text {diss }}=\overline{\overline{\boldsymbol{\eta}}} \boldsymbol{J} \cdot \boldsymbol{J}$. The parameter $f$ therefore determines the fraction of magnetic energy that is radiated away instead of being dissipated locally inside the disk, thereby increasing its entropy. Finally the system of equations is closed by the equation of state of ideal gases $P=n K T$ where $n=\rho / m_{\mathrm{p}}$ ( $m_{\mathrm{p}}$ being the proton mass) is the number density of the gas, $T$ its temperature, and $K$ the Boltzmann constant.

To solve the resistive MHD system of Eqs. (1)-(4), we employ a modified version of the MHD module provided with the public code FLASH $^{1}$ (Fryxell et al. 2000) developed at the ASC FLASH Center at the University of Chicago, adopting its AMR capabilities. The simulations were carried out in 2.5 dimensions, that is, in cylindrical geometry in the coordinates $r$, $z$ assuming axisymmetry around the rotation axis of the diskjet system. The algorithm implemented belongs to the class of high-resolution Godunov schemes that are those best-suited to studying supersonic flows. We therefore used a linear reconstruction of primitive variables with a minmod limiter on pressure and flow speed and a Van Leer limiter on density and on the magnetic field components. Second order accuracy in time is obtained thanks to an Hancock predictor step on the primitive variables, while to compute the fluxes needed to update the conservative variables, we implemented an HLLE solver, which is an approximate linearized Riemann solver that assumes a priori a two-wave configuration for the solution. To control the solenoidality of the magnetic field $(\nabla \cdot \boldsymbol{B}=0)$, the eight-wave approach (Powell et al. 1999) was used, which is known for simply advecting the monopoles, while a parabolic diffusion operator (Marder 1987; see also Dedner et al. 2002) was added to the induction equation to diffuse them. We finally ensured an angular momentum conserving form of the $\phi$ component of the momentum equation Eq. (2) and we used a form of the $\phi$ component of the induction equation Eq. (3) that conserves the poloidal current flux.

\footnotetext{
${ }^{1}$ FLASH is freely available at http://flash.uchicago.edu
}

\subsection{Initial conditions}

In the initial setup of our simulations, we model a disk rotating with a slightly sub-Keplerian speed threaded by an initially purely poloidal magnetic field. The initial disk model is derived by imposing equilibrium between the forces initially intervening inside the disk, namely, gravity, centrifugal force, thermal pressure gradients, and Lorentz force. The disk setup is therefore a solution of the following system of equations:

$$
\begin{aligned}
& \frac{\partial P}{\partial z}=-\rho \frac{\partial \Phi_{\mathrm{g}}}{\partial z}-J_{\phi} B_{r} \\
& \frac{\partial P}{\partial r}=-\rho \frac{\partial \Phi_{\mathrm{g}}}{\partial r}+J_{\phi} B_{z}+\frac{\rho u_{\phi}^{2}}{r} .
\end{aligned}
$$

The system of Eqs. (6)-(7) can be easily solved in separable variables assuming radial self-similarity. With this assumption, all the physical quantities $U$ are given by the product of a power law for $r$ with a function of the variable $x=z / r$

$U=U_{0}\left(\frac{r}{r_{0}}\right)^{\beta_{U}} f_{U}\left(\frac{z}{r}\right)$

where $z=0$ corresponds to the midplane of the disk. In this expression, $f_{U}$ is an even function, such that $f_{U}(0)=1$, or an odd function, such that $f_{U}(0)=0$, depending on the symmetry of the variable with respect to the midplane of the disk. For the physical quantities that have an even symmetry $\left(P, \rho, u_{r}, u_{\phi}, B_{z}\right), U_{0}$ therefore represents their value at $r=r_{0}, z=0$.

Self-similarity requires that all the characteristic speeds, namely sound and Alfvén speed, and flow speeds should scale as the Keplerian velocity $\left(\propto r^{-1 / 2}\right)$ on the midplane of the disk. Also imposing a polytropic relation between the disk density and pressure, that is, $P=P_{0}\left(\rho / \rho_{0}\right)^{\gamma}$, the power law coefficients $\beta_{U}$ are determined as follows:

$$
\begin{aligned}
& \beta_{u_{\phi}}=\beta_{u_{r}}=\beta_{u_{z}}=-1 / 2 \quad \beta_{P}=-5 / 2 \\
& \beta_{B_{r}}=\beta_{B_{z}}=-5 / 4 \quad \beta_{\rho}=-3 / 2 .
\end{aligned}
$$

The initial poloidal magnetic field has been set through the flux function $\Psi$ to ensure the solenoidality of the field:

$\Psi=\frac{4}{3} B_{z 0} r_{0}^{2}\left(\frac{r}{r_{0}}\right)^{3 / 4} \frac{m^{5 / 4}}{\left(m^{2}+z^{2} / r^{2}\right)^{5 / 8}}$.

The components of the field, obtained thanks to the simple relations

$B_{z}=\frac{1}{r} \frac{\partial \Psi}{\partial r} \quad B_{r}=-\frac{1}{r} \frac{\partial \Psi}{\partial z}$

obviously fulfill the self-similarity requirements. The parameter $m$ determines the height scale on which the initial magnetic field bends, where a value $m \rightarrow \infty$ gives a perfectly vertical $\left(B_{r}=0\right)$ field.

Given the poloidal magnetic field, the vertical equilibrium Eq. (6) can be numerically solved to give the vertical profiles of disk density $\left(f_{\rho}\right)$ and pressure $\left(f_{P}=f_{\rho}^{\gamma}\right)$. On the other hand, the radial equilibrium Eq. (7) can be solved to determine the disk rotation speed $u_{\phi}$. The solution will therefore depend on the following non-dimensional parameters:

$\epsilon=\left.\frac{c_{\mathrm{s}}}{V_{\mathrm{K}}}\right|_{z=0}=\left.\sqrt{\frac{p}{\rho} \frac{r}{G M}}\right|_{z=0}$,

which is the ratio between the sound speed $c_{\mathrm{s}}=\sqrt{P / \rho}$ and the Keplerian rotation speed $V_{\mathrm{K}}=\sqrt{G M / r}$ evaluated on the disk 
midplane. This quantity determines the disk thermal height scale $H$ through the relation $H=\epsilon r$ (see Frank et al. 2002; Ferreira \& Pelletier 1995). The magnetization parameter is defined as

$\mu=\left.\frac{B^{2}}{2 P}\right|_{z=0}$,

which gives the ratio between the magnetic and thermal pressure evaluated on the disk midplane. The disk rotation velocity at the midplane is slightly sub-Keplerian, with the deviation depending on the parameters $(\epsilon, \mu$, and $m)$ :

$\left.u_{\phi}\right|_{z=0}=\left[1-\frac{5}{2} \epsilon^{2}-2 \epsilon^{2} \mu\left(\frac{5}{4}+\frac{5}{3 m^{2}}\right)\right] \sqrt{\frac{G M}{r}}$.

The toroidal velocity decreases towards the disk surface, and it falls to zero where the radial Lorentz force balances the gravitational pull and the thermal pressure gradient.

For the components of the magnetic diffusivity tensor $\overline{\bar{\eta}}$ acting in the disk, we adopt an $\alpha$ prescription (Shakura \& Sunyaev 1973 ) in the same vein as Ferreira (1997 and related works) and Casse \& Keppens $(2002,2004)$. Despite being a common way to parametrize the transport coefficients inside an accretion disk, the origin of this anomalous diffusivity is still being debated. One of the most promising hypotheses states that the anomalous transport has a turbulent origin, triggered by some disk instability, the magneto-rotational instability (MRI, Balbus \& Hawley 1998) the most accredited one. The $\eta_{\phi \phi}=\eta_{\mathrm{m}}$ component is parametrized as follows:

$\eta_{\mathrm{m}}=\left.\alpha_{\mathrm{m}} \quad V_{\mathrm{A}}\right|_{z=0} H \exp \left(-2 \frac{z^{2}}{H^{2}}\right)$,

where $\alpha_{\mathrm{m}}$ is a constant parameter, $\left.V_{\mathrm{A}}\right|_{z=0}=\left.\left(B_{z} / \sqrt{\rho}\right)\right|_{z=0}$ is the Alfven speed calculated on the disk midplane, and $H=$ $\left.\left(c_{\mathrm{S}} / \Omega_{\mathrm{K}}\right)\right|_{z=0}$ is the thermal height scale of the disk. In the simulations, both the Alfven speed and $H$ are allowed to evolve in time. The other components of the diffusivity tensor $\eta_{r r}=\eta_{z z}=\eta_{\mathrm{m}}^{\prime}$ are assumed to be proportional to $\eta_{\mathrm{m}}$ through an anisotropy parameter $\chi_{\mathrm{m}}$, which is the inverse of the analogous parameter introduced by Ferreira \& Pelletier (1995):

$\chi_{\mathrm{m}}=\frac{\eta_{\mathrm{m}}^{\prime}}{\eta_{\mathrm{m}}}$

A ratio $\chi_{\mathrm{m}}=1$ indicates an isotropic resistive configuration. We recall that the presence of an effective resistivity inside the disk allows the magnetic field to break the "frozen-in" condition and the matter to slip through the field lines. The component $\eta_{\mathrm{m}}$, therefore indicated as poloidal resistivity, allows the flow to slip through the field in the poloidal plane while $\eta_{\mathrm{m}}^{\prime}$, indicated as toroidal resistivity, controls the diffusion of the toroidal component of the field.

In a steady situation the accretion motion should be consistent with the poloidal magnetic field configuration, as stated by the poloidal induction equation in its stationary form

$u_{z} B_{r}-u_{r} B_{z}=\eta_{\mathrm{m}} J_{\phi}$

which expresses the balance between the advection and the diffusion of the poloidal magnetic field. Therefore we initially impose an accretion flow inside the disk solving Eq. (15) with the condition $u_{z}=\frac{z}{r} u_{r}$. Due to the self-similarity requirements, the radial accretion speed scales initially as the Keplerian speed on the midplane of the disk:

$\left.u_{r}\right|_{z=0}=-\alpha_{\mathrm{m}}(2 \mu)^{1 / 2} \epsilon^{2}\left(\frac{5}{4}+\frac{5}{3 m^{2}}\right) \sqrt{\frac{G M}{r}}$.

As the poloidal resistivity $\eta_{\mathrm{m}}$ exponentially decreases towards the disk surface, the initial accretion flow cancels out outside the disk.

It must be pointed out that in the initial conditions, since there is no toroidal magnetic field, there is no mechanism of angular momentum transport that can support the initial accretion flow: the angular momentum transport associated with the toroidal field will be triggered by torsional Alfvén waves due to the differential rotation between the midplane and the surface of the disk. Moreover, since we will assume a magnetic field around equipartition with the thermal energy on the midplane of the disk (see Sect. 2.5), the time scale on which the transport of angular momentum will become effective, given by the Alfvén crossing time of the disk thickness, is comparable to the local period of rotation of the Keplerian disk and to its epicyclic frequency.

On top of the disk we prescribe a hydrostatic, spherically symmetric atmosphere for which we impose, as for the disk, a polytropic relation between pressure $P_{\mathrm{a}}$ and density $\rho_{\mathrm{a}}: P_{\mathrm{a}}=$ $P_{\mathrm{a} 0}\left(\rho_{\mathrm{a}} / \rho_{\mathrm{a} 0}\right)^{\gamma}$. The density and pressure distribution are therefore given by

$\rho_{\mathrm{a}}=\rho_{\mathrm{a} 0}\left(\frac{r_{0}}{R}\right)^{\frac{1}{\gamma-1}} \quad P_{\mathrm{a}}=\rho_{\mathrm{a} 0} \frac{\gamma-1}{\gamma} \frac{G M}{r_{0}}\left(\frac{r_{0}}{R}\right)^{\frac{\gamma}{\gamma-1}}$,

where $\rho_{\mathrm{a} 0}$ is the value of the atmosphere density at the spherical radius $R=r_{0}$. The initial position of the disk surface is located where the disk and atmosphere pressures are equal. The initial position of the disk surface is therefore determined by its temperature, defined by $\epsilon$, by the field intensity $\mu$, its inclination, given by the parameter $m$, and by the density contrast between the disk and the corona $\rho_{\mathrm{a} 0} / \rho_{0}$.

\subsection{Units and normalization}

Since in the formulation of the problem we did not introduce any specific physical scale, the system of Eqs. (1)-(4) and the initial conditions presented in Sect. 2.2 can be normalized in arbitrary units. The results will be therefore presented in non-dimensional units.

Lengths will be given in units of $r_{0}$, corresponding approximatively to the inner truncation radius of the disk. Speeds will be expressed in units of the Keplerian speed $V_{\mathrm{K} 0}=\sqrt{G M / r_{0}}$ at $r=r_{0}$, and the densities in units of $\rho_{0}$, which is the disk initial density at $r=r_{0}, z=0$. Assuming for $r_{0}$ the following units, appropriated for YSO or AGN systems,

$$
\begin{aligned}
r_{0} & =0.1 \mathrm{AU} \quad(\mathrm{YSO}) \\
& =10 R_{\mathrm{Schw}}=10^{-4}\left(\frac{M}{10^{8} M_{\odot}}\right) \mathrm{pc} \quad(\mathrm{AGN})
\end{aligned}
$$

where $R_{\text {Schw }}=2 G M / c^{2}$ is the Schwarzschild radius, the Keplerian speed $V_{\mathrm{K} 0}$ is given by

$$
\begin{aligned}
V_{\mathrm{K} 0} & =94\left(\frac{M}{M_{\odot}}\right)^{1 / 2}\left(\frac{r_{0}}{0.1 \mathrm{AU}}\right)^{-1 / 2} \mathrm{~km} \mathrm{~s}^{-1} \\
& =6.7 \times 10^{4}\left(\frac{r_{0}}{10 R_{\mathrm{Schw}}}\right)^{-1 / 2} \mathrm{~km} \mathrm{~s}^{-1}
\end{aligned}
$$


Correspondingly, time is expressed in units of $t_{0}=r_{0} / V_{\mathrm{K} 0}$ :

$$
\begin{aligned}
t_{0} & =1.7\left(\frac{M}{M_{\odot}}\right)^{-1 / 2}\left(\frac{r_{0}}{0.1 \mathrm{AU}}\right)^{3 / 2} \text { days } \quad(\mathrm{YSO}) \\
& =0.5\left(\frac{M}{10^{8} M_{\odot}}\right)\left(\frac{r_{0}}{10 R_{\mathrm{Schw}}}\right)^{3 / 2} \text { days } \quad(\mathrm{AGN})
\end{aligned}
$$

Expressed in units of $t_{0}$, the Keplerian period of rotation at the inner radius of the disk $r_{0}$ is equal to $2 \pi$.

Finally the normalization density $\rho_{0}$ can be chosen by determining a suitable mass accretion rate unit $\dot{M}_{0}=r_{0}^{2} \rho_{0} V_{\mathrm{K} 0}$ :

$$
\begin{aligned}
\dot{M}_{0} & =3 \times 10^{-7}\left(\frac{\rho_{0}}{10^{-12} \mathrm{~g} \mathrm{~cm}^{-3}}\right)\left(\frac{M}{M_{\odot}}\right)^{1 / 2}\left(\frac{r_{0}}{0.1 \mathrm{AU}}\right)^{3 / 2} M_{\odot} \mathrm{yr}^{-1} \\
& =9\left(\frac{\rho_{0}}{10^{-12} \mathrm{~g} \mathrm{~cm}^{-3}}\right)\left(\frac{M}{10^{8} M_{\odot}}\right)^{2}\left(\frac{r_{0}}{10 R_{\mathrm{Schw}}}\right)^{3 / 2} M_{\odot} \mathrm{yr}^{-1}(21
\end{aligned}
$$

where, as customary, the first expression corresponds to YSO and the second to AGN objects. The values of accretion and outflow mass rates will be presented in non-dimensional units, and they must be multiplied by the $\dot{M}_{0}$ factor of Eq. (21) to obtain their physical value. Similarly torques and powers that are shown subsequently will be given in units of $\dot{J}_{0}=r_{0}^{3} \rho_{0} V_{\mathrm{K} 0}^{2}$ and $\dot{E}_{0}=r_{0}^{2} \rho_{0} V_{\mathrm{K} 0}^{3}$, respectively:

$$
\begin{aligned}
& \dot{J}_{0}=3 \times 10^{38}\left(\frac{\rho_{0}}{10^{-12} \mathrm{~g} \mathrm{~cm}^{-3}}\right)\left(\frac{M}{M_{\odot}}\right)\left(\frac{r_{0}}{0.1 \mathrm{AU}}\right)^{2} \text { dyne cm } \\
& =1.2 \times 10^{51}\left(\frac{\rho_{0}}{10^{-12} \mathrm{~g} \mathrm{~cm}^{-3}}\right)\left(\frac{M}{10^{8} M_{\odot}}\right)^{3}\left(\frac{r_{0}}{10 R_{\mathrm{Schw}}}\right)^{2} \text { dyne cm }(22)
\end{aligned}
$$

$\dot{E}_{0}=1.9 \times 10^{33}\left(\frac{\rho_{0}}{10^{-12} \mathrm{~g} \mathrm{~cm}^{-3}}\right)\left(\frac{M}{M_{\odot}}\right)^{3 / 2}\left(\frac{r_{0}}{0.1 \mathrm{AU}}\right)^{1 / 2} \mathrm{erg} \mathrm{s}^{-1}$

$=2.6 \times 10^{46}\left(\frac{\rho_{0}}{10^{-12} \mathrm{~g} \mathrm{~cm}^{-3}}\right)\left(\frac{M}{10^{8} M_{\odot}}\right)^{2}\left(\frac{r_{0}}{10 R_{\mathrm{Schw}}}\right)^{1 / 2} \mathrm{erg} \mathrm{s}^{-1}$.

Again, the first equations must be used for YSO while the second for AGN systems.

\subsection{Boundary conditions}

Besides restricting our study to axisymmetric structures, we also assume that the system is symmetric with respect to the midplane of the disk. The computational domain therefore covers a rectangular region with a radial extent $\left[0,40 r_{0}\right]$ and a size along the $z$ direction equal to $\left[0,120 r_{0}\right]$ : axisymmetry is imposed on the rotation axis $r=0$, while planar symmetry is imposed on the disk midplane $z=0$. Besides choosing suitable boundary conditions on the outer sides of the domain, an inner boundary must be placed inside the computational box in order to avoid the singularity of the potential well and of the initial setup at the origin of the axis. We therefore define a rectangular box with a size $r \times z=\left[0, r_{0}\right] \times\left[0,0.5 r_{0}\right]$ that is excluded from the computation and on whose sides boundary conditions must be set.

On the outer right boundary $\left(r=40 r_{0}\right)$, "outflow" conditions, that is zero-gradient, are imposed on thermal pressure, density, and poloidal velocity components. On one disk's thermal height scale $(z<H r)$, the value of the radial velocity in the ghost zones is set to the extrapolated value only if negative and zero otherwise. In this way we avoid any outflow of matter without imposing the mass accretion rate, which will be determined by the dynamical evolution of the system. The continuity of the first derivative is required for $u_{\phi}, B_{z}, B_{\phi}$ too. The condition on $B_{r}$ is determined by imposing the solenoidality of the field, $\nabla \cdot \boldsymbol{B}=0$, on the last cell of the domain with a firstorder approximation. On the outer upper boundary $\left(z=120 r_{0}\right)$, "outflow" conditions are imposed on all the variables except for $B_{z}$ whose boundary values are determined to satisfy the $\nabla \cdot \boldsymbol{B}=0$ requirement.

We decided to prescribe the continuity of the first derivative of some variables in the radial direction since the power-law behavior of the initial conditions along $r$ (see Eq. (8)) determines strong gradients of the physical quantities on the rightmost boundary. This boundary condition above all affects the radial component of the Lorentz force $F_{r}$

$F_{r}=-\frac{1}{2} \frac{\partial B_{\phi}^{2}}{\partial r}-\frac{B_{\phi}^{2}}{r}-\frac{1}{2} \frac{\partial B_{z}^{2}}{\partial r}+B_{z} \frac{\partial B_{r}}{\partial z}$

and therefore the collimation of the outflow. First of all, it is easy to see that a zero-gradient condition on the toroidal magnetic field component $B_{\phi}$ cancels the pressure gradient of the magnetic pressure (first term on the right hand side of Eq. (24)) while the pinching force $-B_{\phi}^{2} / r$ (second one) is still present. An outflow condition on $B_{\phi}$ on the right outer boundary thus enforces the collimation due to the toroidal field. This issue has been widely discussed by Ustyugova et al. (1999) who proposed to use a "force-free" condition, where the poloidal electrical current is parallel to the magnetic field, in order to remove artificial forces originating at the boundaries. Seen from the point of view of electrical currents, a zero-gradient condition on $B_{\phi}$ corresponds to having a negative collimating current component $J_{z}$ flowing along the right boundary, while stationary models of accretion-ejection structures show that, in the outer part of the disk, the current flows out from it and pushes the flow along the field lines without collimating it (see Fig. 13 in Ferreira 1997). The continuity of the first derivative of $B_{\phi}$ at the rightmost boundary allows a gradient of magnetic pressure associated with $B_{\phi}$ to develop, which can counteract the toroidal pinch and generate an outflowing positive current $J_{z}$.

Even if the effects are less pronounced, the continuity of the first derivative of $B_{z}$ on the rightmost boundary also affects the collimation of the structure. A pressure gradient directed outwards associated with $B_{z}$ (third term on the right hand side of Eq. (24)) counteracts the poloidal field tension (fourth term), thus producing slightly less collimated structures.

On the other hand, we noticed that the choice of an "outflow" condition for the toroidal field on the upper boundary did not affect the behavior of the outflow as much as the condition on the right boundary. We did not notice a huge difference between the "outflow" condition, where the poloidal current has only a $z$ component, and the "force-free" condition proposed by Ustyugova et al. (1999), where the poloidal current is parallel to the poloidal field.

At the inner boundaries located on the edges of the rectangular region $r \times z=\left[0, r_{0}\right] \times\left[0,0.5 r_{0}\right]$, we adopted a similar strategy. On the $r=r_{0}$ side we extrapolated all the physical quantities imposing the continuity of the first derivative except for the poloidal components of the velocity field, for which a zerogradient condition was used, and for the radial component of the magnetic field, which is required to fulfill the $\nabla \cdot \boldsymbol{B}=0$ condition. On the $z=0.5 r_{0}$ side we imposed an "outflow" condition on all the variables except for the $z$ component of the field, determined by imposing the solenoidality of the field. All the details on the simulation performed are given in the next section. 
Table 1. Initial parameters of the simulations.

\begin{tabular}{cccccccc}
\hline \hline Simulation & $\alpha_{\mathrm{m}}$ & $\chi_{\mathrm{m}}$ & $f$ & $\dot{M} / \dot{M}_{0}$ & $\dot{J} / \dot{J}_{0}$ & $\dot{E} / \dot{E}_{0}$ & Resolution \\
\hline 1 & 0.1 & 1 & 1 & $7.3 \times 10^{-3}$ & $1.4 \times 10^{-2}$ & $3.3 \times 10^{-3}$ & $512 \times 1536$ \\
2 & 0.1 & 1 & 1 & $7.3 \times 10^{-3}$ & $1.4 \times 10^{-2}$ & $3.3 \times 10^{-3}$ & $128 \times 384$ \\
3 & 0.1 & 1 & 0 & $7.3 \times 10^{-3}$ & $1.4 \times 10^{-2}$ & $3.3 \times 10^{-3}$ & $512 \times 1536$ \\
4 & 1 & 1 & 1 & $7.3 \times 10^{-2}$ & $1.4 \times 10^{-1}$ & $3.3 \times 10^{-2}$ & $512 \times 1536$ \\
5 & 1 & 3 & 1 & $7.3 \times 10^{-2}$ & $1.4 \times 10^{-1}$ & $3.3 \times 10^{-2}$ & $512 \times 1536$ \\
6 & 1 & 3 & 0 & $7.3 \times 10^{-2}$ & $1.4 \times 10^{-1}$ & $3.3 \times 10^{-2}$ & $512 \times 1536$ \\
\hline
\end{tabular}

\subsection{The simulations}

Once they are normalized with the units given in Sect. 2.3, the initial conditions presented in Sect. 2.2 depend on 6 nondimensional parameters: the ratio between the sound speed and the Keplerian speed at the disk midplane $\epsilon$, the disk magnetization parameter $\mu$, the magnetic height scale of the initial field $m$, the strength of magnetic diffusivity $\alpha_{\mathrm{m}}$ and its anisotropy coefficient $\chi_{\mathrm{m}}$, the ratio between the initial atmosphere and disk densities $\rho_{\mathrm{a} 0} / \rho_{0}$. All the free parameters except those describing the diffusive properties of the disk will be the same for all the simulations. We therefore assume a parameter $\epsilon=0.1$, which fixes the initial thermal height scale and temperature $T$ on the midplane of the disk, that is,

$$
\begin{aligned}
\left.T\right|_{z=0}=\epsilon^{2} \frac{m_{\mathrm{p}} G M}{K r} & =10^{4}\left(\frac{\epsilon}{0.1}\right)^{2}\left(\frac{M}{M_{\odot}}\right)\left(\frac{r}{0.1 \mathrm{AU}}\right)^{-1} \mathrm{~K} \\
& =5 \times 10^{9}\left(\frac{\epsilon}{0.1}\right)^{2}\left(\frac{r}{10 R_{\mathrm{Schw}}}\right)^{-1} \mathrm{~K} .
\end{aligned}
$$

The initial structure of the magnetic field is determined by fixing the magnetization parameters $\mu=0.3$ and $m=0.35$, which therefore gives a magnetic height scale 3.5 times higher than the initial thermal height scale of the disk. We recall that a value of magnetization around equipartition or slightly below it is generally required both in numerical (see Zanni et al. 2004; Casse \& Keppens 2002) and analytical (Ferreira 1997; Casse \& Ferreira 2000a) modeling of accretion disks launching jets. This condition is determined by the equilibrium between thermal pressure gradients, which vertically support the disk and are responsible for the initial mass loading the field lines, and Lorentz forces, which tend to pinch the disk. The magnetization parameter $\mu$ fixes the initial value of the poloidal magnetic field on the disk midplane:

$$
\begin{aligned}
& \left.B\right|_{z=0}=\sqrt{\mu 8 \pi P} \\
& =2.6\left(\frac{\mu}{0.3}\right)^{1 / 2}\left(\frac{\epsilon}{0.1}\right)\left(\frac{\rho_{0}}{10^{-12} \mathrm{~g} \mathrm{~cm}^{-3}}\right)^{1 / 2}\left(\frac{M}{M_{\odot}}\right)^{1 / 2}\left(\frac{r}{0.1 \mathrm{AU}}\right)^{-5 / 4} \mathrm{G} \\
& =1.8 \times 10^{3}\left(\frac{\mu}{0.3}\right)^{1 / 2}\left(\frac{\epsilon}{0.1}\right)\left(\frac{\rho_{0}}{10^{-12} \mathrm{~g} \mathrm{~cm}^{-3}}\right)^{1 / 2}\left(\frac{r}{10 R_{\text {Schw }}}\right)^{-5 / 4} \mathrm{G} .(26)
\end{aligned}
$$

Finally we assumed a density ratio between the corona and the $\operatorname{disk} \rho_{\mathrm{a} 0} / \rho_{0}=10^{-4}$.

In order to investigate the effects of magnetic resistivity, we performed a series of simulations varying the value of the $\alpha_{\mathrm{m}}$ parameter and the anisotropy factor $\chi_{\mathrm{m}}$. The summary of all the simulations performed is given in Table 1 . From the second to the eighth column, we show the poloidal resistivity parameter $\alpha_{\mathrm{m}}$, the anisotropy of magnetic resistivity $\chi_{\mathrm{m}}$, the cooling parameter $f$, the initial accretion rate $\dot{M} / \dot{M}_{0}$, the torque $\dot{J} / \dot{J}_{0}$ needed to support the accretion rate between $r_{\mathrm{i}}=r_{0}$ and $r_{\mathrm{e}}=10 r_{0}$, the accretion energy $\dot{E} / \dot{E}_{0}$ liberated between the same radii, and the equivalent resolution of the adaptive grid.

Isotropic resistive configurations $\left(\chi_{\mathrm{m}}=1\right)$ have been studied for two different values of the $\alpha_{\mathrm{m}}$ parameter: $\alpha_{\mathrm{m}}=0.1$ (simulation 1), which corresponds to the magnetic resistivity adopted by Casse $\&$ Keppens $(2002,2004)$, and $\alpha_{\mathrm{m}}=1$ (simulation 4$)$. To determine the effects of an anisotropic resistivity, required by the steady models of Ferreira \& Pelletier (1995), we performed a simulation characterized by $\alpha_{\mathrm{m}}=1$ and $\chi_{\mathrm{m}}=3$ (simulation 5): the parameters $\alpha_{\mathrm{m}}, \chi_{\mathrm{m}}, \mu$, and $\epsilon$ of this simulation are typical of the cold self-similar solutions found by Casse $\&$ Ferreira (2000a).

For these standard simulations, the adaptive mesh provided with FLASH is set up with 7 levels of refinement based on blocks of $8 \times 8$ square cells, giving an equivalent resolution of $512 \times$ 1536 points, which is kept fixed in the disk region $(z<3 \epsilon r)$ and around the inner boundary rectangle, while the grid is free to change and adjust the resolution in the outflow region. In order to determine the importance of numerical diffusive effects in our simulations, we repeated the case characterized by an isotropic $\alpha_{\mathrm{m}}=0.1$ with a resolution four times lower than the usual one, thus increasing the numerical dissipative effects (simulation 2). For this simulation the adaptive grid is allowed to reach a maximum equivalent resolution of $128 \times 384$ points, while maintaining the higher one $(512 \times 1536$ points $)$ around the central inner boundary. The resolution of this test case is similar to the one adopted by Casse \& Keppens (2002, 2004).

This first set of four simulations is characterized by a cooling factor $f=1$ (Eq. (5)), which implies that all the magnetic energy Ohmically dissipated is radiated away. We therefore repeated simulations 1 and 5 assuming a cooling factor $f=0$ to study the effects of the Ohmic heating in the case of a lower (simulation 3) and higher resistivity (simulation 6). The results presented in Sects. 3-7 will refer to the "cold" cases characterized by $f=1$, while a few comments on the "heated" $f=0$ simulations will be done in Sect. 8.

In the fifth column of Table 1 we also show the mass accretion rate that we imposed at the beginning of our simulations, recalling that this initial rate is determined by balancing diffusion and advection of the poloidal field inside the disk. Obviously, energy and angular momentum must be extracted from the accretion flow to support this accretion rate: in the sixth and seventh columns of the same table we also show the torque $\dot{J}$ and the power $\dot{E}$ liberated in the accretion between $r_{\mathrm{i}}=r_{0}$ and $r_{\mathrm{e}}=10 r_{0}$. The values of $\dot{J}$ and $\dot{E}$ are given with a good approximation by:

$$
\begin{aligned}
& \dot{J}=\dot{M}\left(\sqrt{G M r_{\mathrm{e}}}-\sqrt{G M r_{\mathrm{i}}}\right) \\
& \dot{E}=\dot{M}\left(\frac{G M}{2 r_{\mathrm{i}}}-\frac{G M}{2 r_{\mathrm{e}}}\right)
\end{aligned}
$$



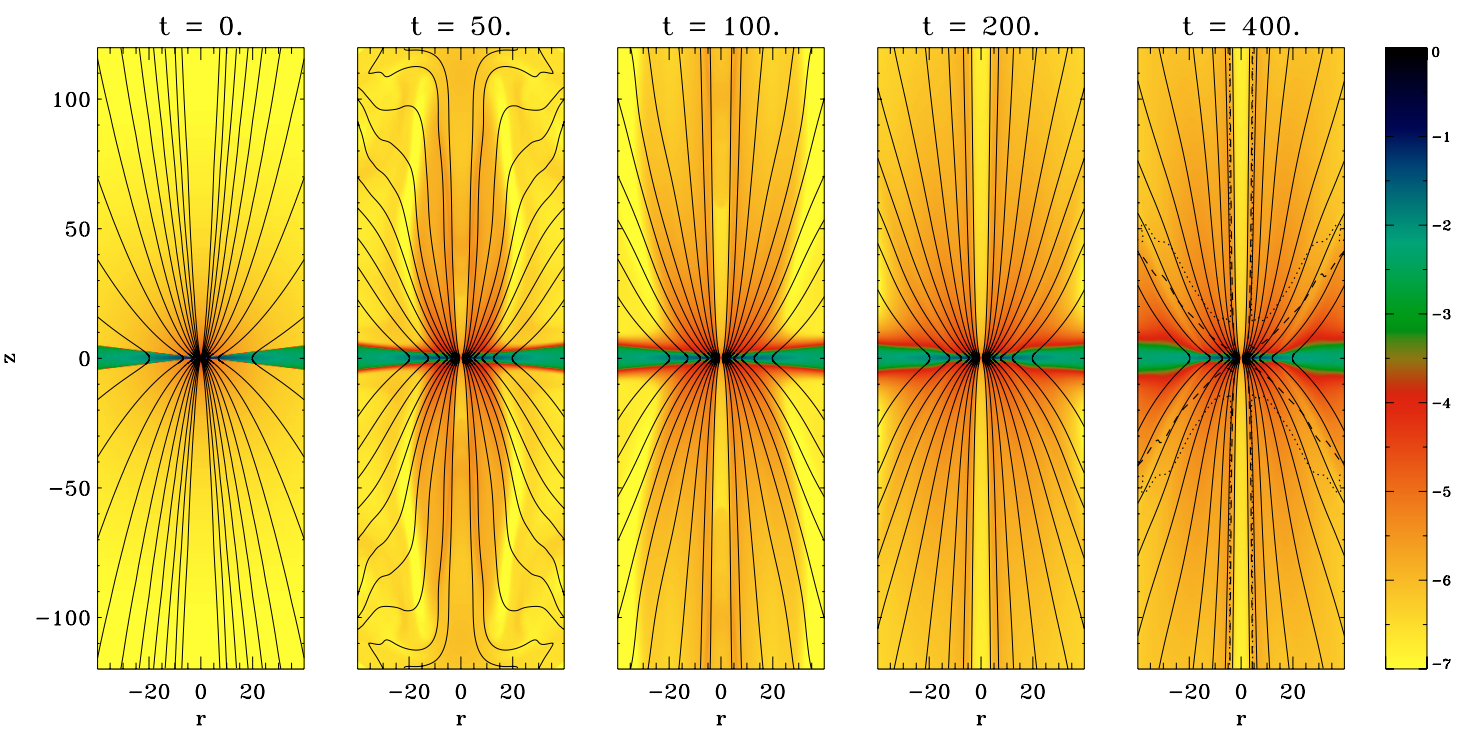

Fig. 1. Time evolution of density maps (in logarithmic scale) of the simulation characterized by $\left(\alpha_{\mathrm{m}}=1, \chi_{\mathrm{m}}=3, f=1\right)$. Time is given in units of $t_{0}$ (see text). In these units the Keplerian period at the inner radius of the disk $r=r_{0}$ is equal to $2 \pi$. Superimposed are sample magnetic field lines: the distance between the field lines is proportional to the intensity of the field. In the last panel $(t=400)$ the critical Alfvén (dashed line) and fast-magnetosonic (dotted line) surfaces are also plotted.
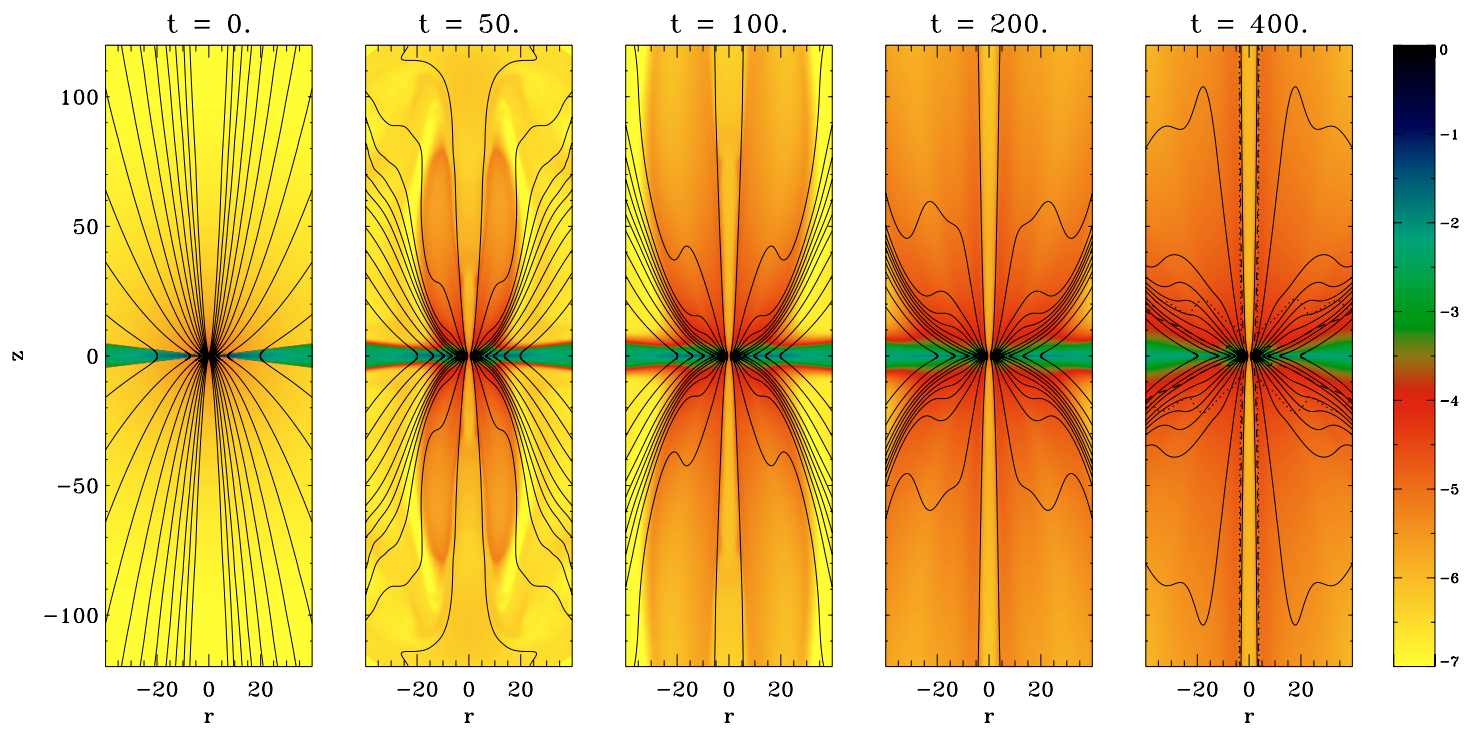

Fig. 2. Same as Fig. 1 but for the case characterized by $\left(\alpha_{\mathrm{m}}=0.1, \chi_{\mathrm{m}}=1, f=1\right)$.

\section{Production of jets from magnetized accretion disks}

All the simulations were carried on up to a time $t=400$ that corresponds to $\sim 63$ periods of rotation of the disk at its inner radius. On the other hand, the final time $t=400$ corresponds to only 0.25 rotations at the outer boundary $r=40 r_{0}$. It is very unlikely that the outer part of the disk has reached an equilibrium stage at the end of the simulation. Therefore the study of the accretion-ejection system will be restricted mainly to the inner part of the disk $\left(r<10 r_{0}\right.$, see Sect. 4) and to the outflow coming from it. In all the cases studied we observed a robust outflow emerging from the underlying accretion disk, and the solutions show a hollow jet, where the central hole corresponds to the "sink" region $r<r_{0}$. The outflows are not completely collimated at the end of the runs; that is, some matter is flowing out from the computational box from the outer cylinder at $r=40 r_{0}$. Nevertheless in all the solutions, the part of the outflow coming from the inner part of the disk crosses the Alfvénic and fast-magnetosonic critical surfaces inside the domain (see Figs. 1 and 2). Since no disturbance produced in the super-fast region of the outflow can propagate upstream towards the accretion disk, this condition obviously ensures that the outer boundary conditions do not affect the launching region of this part of the outflow. On the other hand, perturbations can still propagate in a transversal direction to the magnetic field lines: if the fastMach cones at the boundaries intersect the computational box, the boundary conditions can still affect the radial structure and therefore the collimation of the outflow (see Ustyugova et al. 1999), as already discussed in Sect. 2.4. To overcome the problem of the influence of boundary conditions on the launching phase, Krasnopolsky et al. (2003) and Anderson et al. (2004) restrict the launching of the wind to a narrow region of the inner accretion disk and choose an initial magnetic configuration so as to contain the entire fast-magnetosonic critical surface inside the computational domain. The closed shape of the critical surfaces 
observed in the cited papers characterizes those solutions more as "X-winds" (Shu et al. 1994), which are wide-angle outflows from the corotation radius of a stellar magnetosphere, while the almost conical shape of the critical surfaces of our solutions is typical of extended disk-winds. Moreover, on one hand it cannot be taken for granted that an underlying accretion disk can support the magnetic structure and provide the mass load imposed as a boundary condition by the authors; on the other hand, it has been shown (Ferreira et al. 2006) that "X-winds" have kinematic properties that are inconsistent with observations of $\mathrm{T}$ Tauri microjets.

The structure of the magnetic field allows two classes of solutions to be distinguished. The cases characterized by $\alpha_{\mathrm{m}}=1$ show an "ordered" magnetic configuration at the end of the computation (Fig. 1), while the poloidal field lines are strongly warped and distorted if $\alpha_{\mathrm{m}}=0.1$ (Fig. 2). An exception is represented by the case performed with a lower resolution, since it does not present the characteristic field inversions despite having a small resistivity parameter $\alpha_{\mathrm{m}}=0.1$ (Fig. 3). This anomalous behavior can be reasonably ascribed to the higher numerical dissipation determined by the lower resolution.

Other differences can be noticed in Figs. 1 and 2: in the more diffusive case the jet is asymptotically less dense than in the less diffusive one. Both outflows become super-Alfvenic and superfast-magnetosonic, but in the $\alpha_{\mathrm{m}}=0.1$ simulation both characteristic surfaces lie closer to the disk.

Despite these morphological differences between the more and less dissipative cases, the mechanism that drives the outflows from the disk is qualitatively the same. In the following section we make a few general considerations about the forces that determine both the accretion and the ejection flow.

\subsection{Acceleration mechanism}

Since the accretion-ejection mechanism is mainly magnetically driven, it is worth explicitly writing the Lorentz forces acting on the disk-jet system. Following Ferreira (1997), we decompose the Lorentz force $\boldsymbol{F}=\boldsymbol{J} \times \boldsymbol{B}$ in the directions parallel and perpendicular to the poloidal field:

$$
\begin{aligned}
& F_{\phi}=\frac{B_{\mathrm{p}}}{r} \nabla_{\|}\left(r B_{\phi}\right) \\
& F_{\|}=-\frac{B_{\phi}}{r} \nabla_{\|}\left(r B_{\phi}\right) \\
& F_{\perp}=-\frac{B_{\phi}}{r} \nabla_{\perp}\left(r B_{\phi}\right)+J_{\phi} B_{\mathrm{p}},
\end{aligned}
$$

where $B_{\mathrm{p}}$ is the poloidal magnetic field, while $\nabla_{\|}$and $\nabla_{\perp}$ indicate the derivatives parallel and perpendicular to the poloidal field, respectively. These expressions show clearly that the poloidal component of the Lorentz force associated with the toroidal field is perpendicular to the isosurfaces $r B_{\phi}=$ const., which are the surfaces along which the poloidal electric current flows. Moreover, they show that the component parallel to the poloidal field $F_{\|}$and the toroidal one $F_{\phi}$ are linked by the simple relation (see also Casse \& Keppens 2002, 2004):

$F_{\|}=-\frac{B_{\phi}}{B_{\mathrm{p}}} F_{\phi}$

(notice that the toroidal field $B_{\phi}$ assumes negative values in our simulations). This clearly shows that a poloidal current-field configuration that accelerates the outflow along the field lines $\left(F_{\|}>0\right)$ is also accelerating the plasma in the toroidal direction $\left(F_{\phi}>0\right)$ thus providing an additional centrifugal force. This

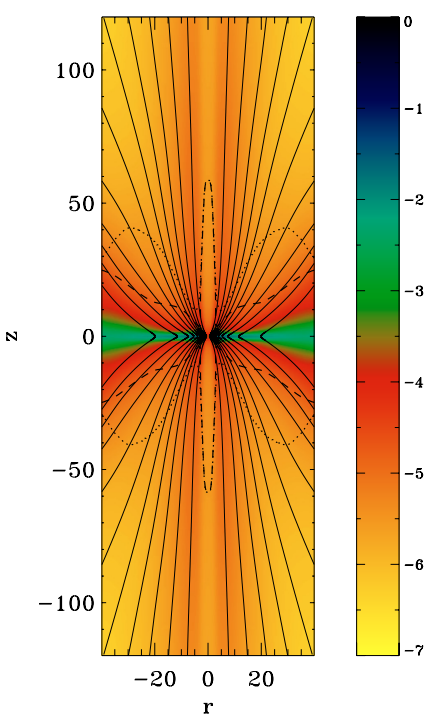

Fig. 3. Density map (logarithmic scale) at $t=400$ of the same simulation shown in Fig. 2 performed with four times smaller resolution.

represents the core of the so-called magneto-centrifugal mechanism, where the magnetic energy stored in the toroidal field at the base of the outflow both accelerates the plasma along the field lines and increases its angular momentum, thus providing a centrifugal acceleration. As stated by the above relation, the relative importance of the two mechanisms, poloidal and centrifugal acceleration, is given by the ratio $\left|B_{\phi}\right| / B_{\mathrm{p}}$. When the toroidal field is stronger than the poloidal one, the gradient of $B_{\phi}$ along the field lines is the main accelerating mechanism; instead, if $\left|B_{\phi}\right| / B_{\mathrm{p}}<1$ the plasma tends to corotate with the mainly poloidal magnetic field and the centrifugal force is dominant.

The conditions are reversed inside the accretion disk. As in a unipolar inductor, a radial electric current develops inside the conducting plasma, thus providing a toroidal force $-J_{r} B_{z}$ that slows the rotation down. According to Eq. (30), since the Lorentz force brakes the matter in the toroidal direction, a poloidal pinching also occurs. Therefore, since the disk is vertically pinched by the gravity, as well as by the magnetic pressure, only the thermal pressure gradient can ensure a quasi-static vertical equilibrium, gently lifting the accreting matter towards the surface of the disk where it can be accelerated to form the outflow. As pointed out in Ferreira (1997), the transition from accretion to ejection can therefore be achieved if the sign of the magnetic torque $F_{\phi}$ changes sign at the disk surface in order to provide a magnetic acceleration.

This scenario is confirmed by our simulations. In Fig. 4 we plot, at the end of our simulations, the total force, given by the sum of gravity, Lorentz force, and thermal pressure gradient, acting along the $z$ direction on the disk height scale. These curves are obtained by averaging inside the region $r_{0}<r<10 r_{0}$ for the cases $\left(\alpha_{\mathrm{m}}=1, \chi_{\mathrm{m}}=3, f=1\right),\left(\alpha_{\mathrm{m}}=1, \chi_{\mathrm{m}}=1, f=1\right)$ and $\left(\alpha_{\mathrm{m}}=0.1, \chi_{\mathrm{m}}=1, f=1\right)$. Along each line we also indicate the location of the points where the magnetic torque and the total $z$-component of the Lorentz force change sign. It is possible to notice that in all the solutions shown, the total force changes sign when the disk is still pinched and braked by the magnetic field so it is the vertical thermal pressure gradient that provides the first vertical acceleration turning the accretion motion into an outflow. The magnetocentrifugal mechanism becomes effective only in correspondence of the triangles, where the plasma is 


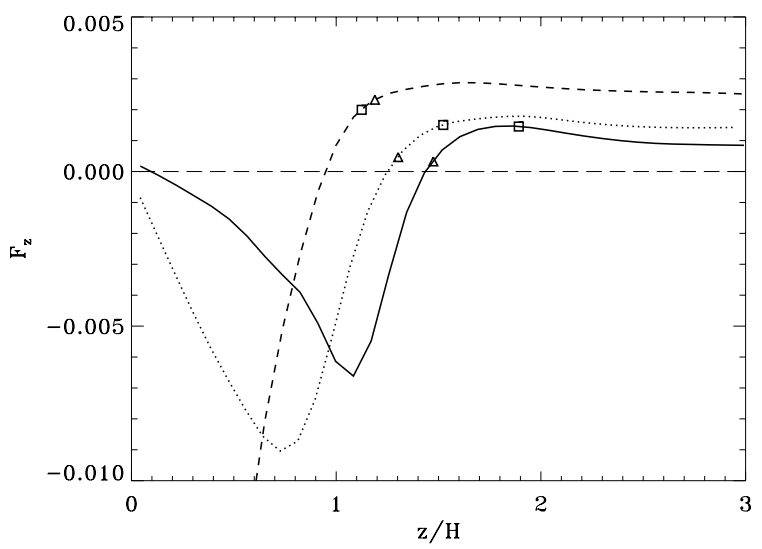

Fig. 4. Total force acting on the disk height scale along the $z$ direction for the cases $\left(\alpha_{\mathrm{m}}=1, \chi_{\mathrm{m}}=3, f=1\right.$, solid line $),\left(\alpha_{\mathrm{m}}=1, \chi_{\mathrm{m}}=1\right.$, $f=1$, dotted line $)$ and $\left(\alpha_{\mathrm{m}}=0.1, \chi_{\mathrm{m}}=1, f=1\right.$, dashed line $)$. Along the curves the points are also indicated where the magnetic torque (triangles) and the $z$ component of the Lorentz force(squares) change sign. The curves are calculated at $t=400$.

accelerated both in the toroidal direction and along the poloidal field lines.

Significant differences can be noticed between the three cases shown: decreasing the value of the poloidal or the toroidal magnetic diffusivity the location of the points where the total force and the magnetic torque change their sign are located at lower height scales. A possible explanation for this behavior can be found in Ferreira (1997) who shows that to change the sign of the magnetic torque it is in fact necessary that the radial current $J_{r}$ responsible for the braking of the disk decreases vertically on a disk scale height. It has been shown that in a stationary situation the radial current falls off on a height scale proportional to $\sqrt{\alpha_{\mathrm{m}}^{2} \chi_{\mathrm{m}}}$ (see Eq. (B1) in Ferreira 1997). Consistent with our simulations, the change of sign of the torque therefore occurs at a higher $z / H$ when the value of $\alpha_{\mathrm{m}}$ or the anisotropy factor $\chi_{\mathrm{m}}$ are increased. A second noticeable difference is that, while in the two higher diffusivity cases $\left(\alpha_{\mathrm{m}}=1\right)$ the torque changes sign when the disk is still pinched by the poloidal magnetic pressure, it changes above this point in the $\alpha_{\mathrm{m}}=0.1$ simulation. In this case the $z$-component of the Lorentz force provides an additional source of mass loading before the magnetocentrifugal effect becomes effective.

Another important difference between these three simulations is shown in Fig. 5, where we plot a radial average of the ratio between the toroidal and poloidal fields $\left|B_{\phi}\right| / B_{\mathrm{p}}$ along the outflows of the three cases. According to Eq. (30), these curves show that the centrifugal effects are stronger when the magnetic diffusivity is anisotropic, while for a low diffusivity the toroidal field pressure gradient is the dominant accelerating force. Moreover, the increase in this ratio with $z$ shows that, while near the disk surface the centrifugal force is effectively contributing to the acceleration of the outflow, it becomes negligible far from it.

\subsection{Current circuits}

A suitable way to understand how the accretion-ejection mechanism works is to analyze the circulation of the poloidal current in the disk-outflow system. In Fig. 6 we show the poloidal current circuits superimposed on density maps in logarithmic scale at $t=400$. Sample field lines and the poloidal speed vectors are also plotted; the left panel refers to the $\left(\alpha_{\mathrm{m}}=0.1, \chi_{\mathrm{m}}=1\right.$,

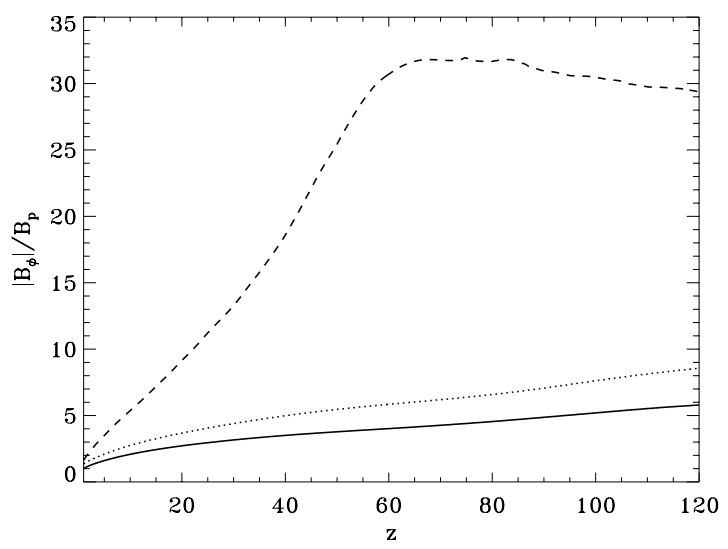

Fig. 5. Average ratio between the toroidal and poloidal magnetic fields along the outflows of the simulations characterized by $\left(\alpha_{\mathrm{m}}=1, \chi_{\mathrm{m}}=3\right.$, $f=1$, solid line $),\left(\alpha_{\mathrm{m}}=1, \chi_{\mathrm{m}}=1, f=1\right.$, dotted line $)$, and $\left(\alpha_{\mathrm{m}}=0.1\right.$, $\chi_{\mathrm{m}}=1, f=1$, dashed line). The curves are calculated at $t=400$.
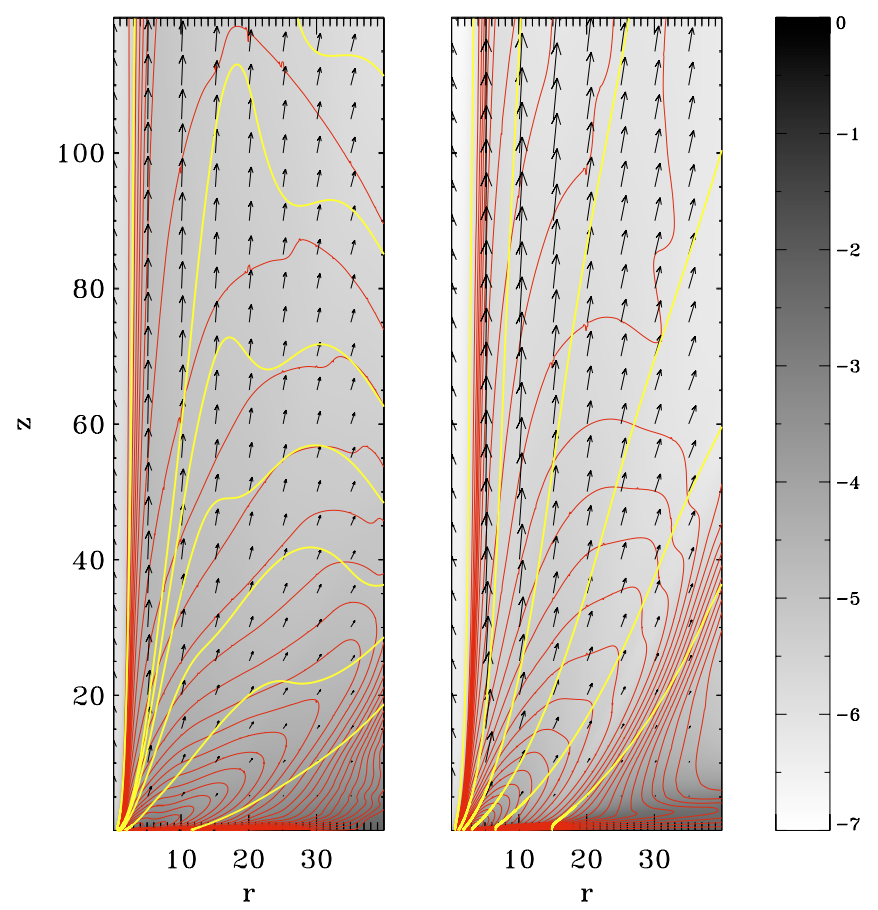

Fig. 6. Poloidal current circuits (solid thin red lines) at $t=400$ of the cases $\left(\alpha_{\mathrm{m}}=0.1, \chi_{\mathrm{m}}=1, f=1\right.$, left panel $)$ and $\left(\alpha_{\mathrm{m}}=1, \chi_{\mathrm{m}}=3\right.$, $f=1$, right line). Plotted are also sample poloidal field lines (solid thick yellow lines) and the poloidal speed vectors. In the background density maps in logarithmic grayscale are shown.

$f=1)$ case and the right one to the $\left(\alpha_{\mathrm{m}}=1, \chi_{\mathrm{m}}=3, f=1\right)$ simulation. The current circuits are given by the $r B_{\phi}=$ const. isosurfaces: the poloidal currents circulate counter-clockwise and the Lorentz forces are directed outwards perpendicular to the closed circuits. As discussed before, a strong radial positive current flows along the disk midplane, thus providing a braking and vertically pinching force. At the disk surface the current changes direction until a force component accelerating the plasma along the poloidal field appears.

Some differences can be noticed between the two panels. Due to a strong advection determined by the accretion flow, the poloidal field lines are much more inclined inside the disk in the less diffusive case (left panel), thus providing a vertically directed magnetic tension which mass loads the outflow as it was 

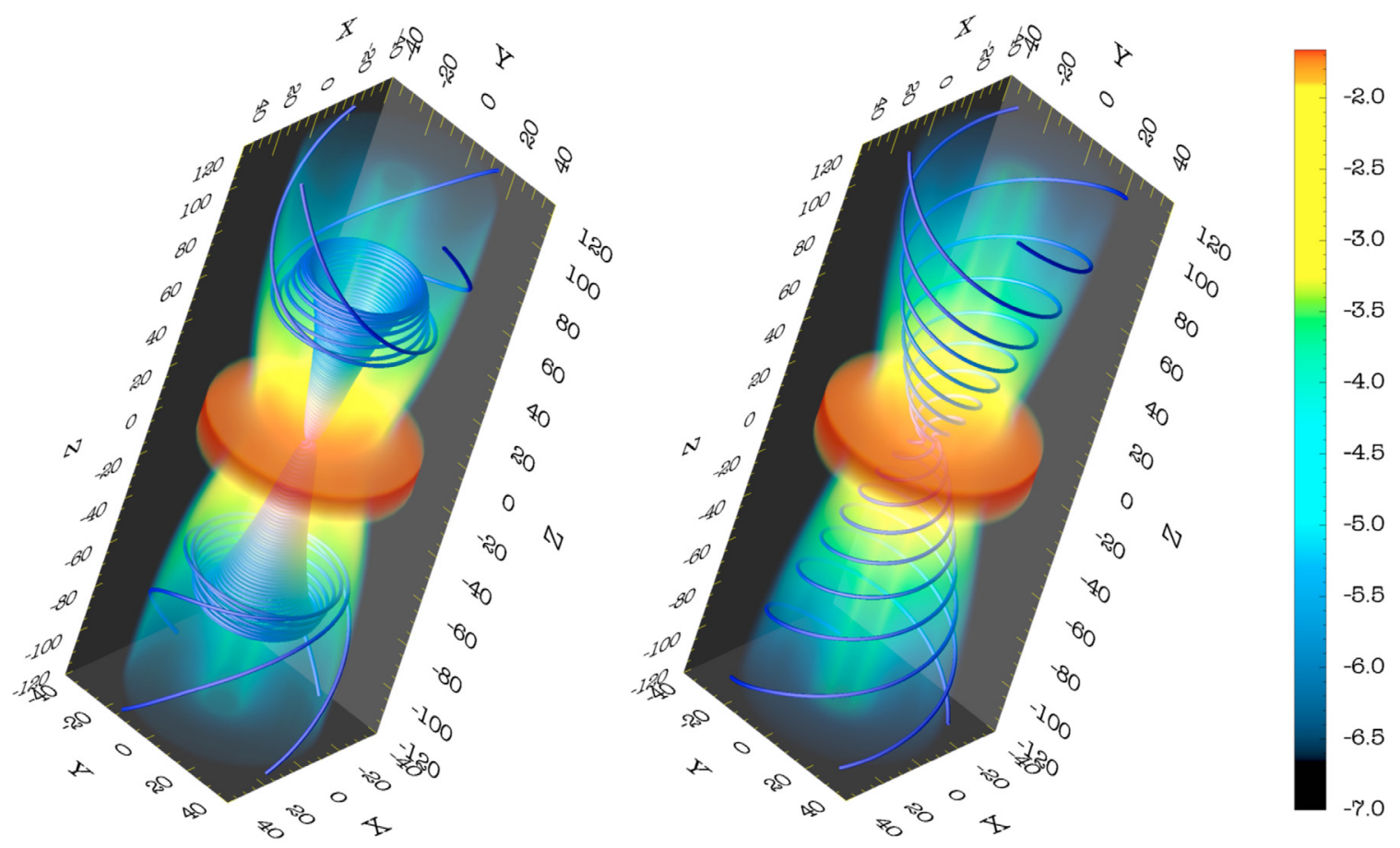

Fig. 7. Three-dimensional rendering of density at $t=130$ for the cases $\left(\alpha_{\mathrm{m}}=0.1, \chi_{\mathrm{m}}=1, f=1\right.$, left panel $)$ and $\left(\alpha_{\mathrm{m}}=1, \chi_{\mathrm{m}}=3, f=1\right.$, right panel). We also plotted four sample magnetic field lines wrapped around the same magnetic surface.

shown in Fig. 4 (see the position of the square symbol along the dashed line). Moreover, it is possible to see that the different shape of the current circuits, together with the higher inclination of the poloidal field lines, develops in the $\alpha_{\mathrm{m}}=0.1 \mathrm{a}$ strong vertical Lorentz force which strongly bends the field lines, as already shown in Fig. 2.

This effect is even more evident if we look at a threedimensional rendering of the two simulations discussed here. In Fig. 7 we show a 3D rendering of density maps in logarithmic scale for the two cases $\left(\alpha_{\mathrm{m}}=0.1, \chi_{\mathrm{m}}=1, f=1\right.$, left panel) and $\left(\alpha_{\mathrm{m}}=1, \chi_{\mathrm{m}}=3, f=1\right.$, right panel) at a time $t=130$. It is possible to see that, in the more diffusive case, the field lines are gently wrapped around the magnetic surfaces. On the other hand, in the $\alpha_{\mathrm{m}}=0.1$ simulation the footpoints of the field lines are advected towards the central object, not balanced by a strong enough diffusion. A strong differential rotation along the field line therefore appears, the footpoint of the line rotating much faster than its opposite end and a strong toroidal field develops at the footpoint, which gives the strong force directed vertically shown in Fig. 6 that completely distorts the field lines. The mainly toroidal feature visible in the left panel of Fig. 7 propagates vertically along the outflow axis similar to a "magnetic tower" (Li et al. 2001; Lynden-Bell 2003). Moreover this mechanism is not transient, repeating itself on every field line when its footpoint is advected towards the faster rotating central part of the disk. Since the $\alpha_{\mathrm{m}}=0.1$ simulation at a low resolution shows a magnetic configuration similar to the right panel of Fig. 7, it is possible to speculate that the higher numerical diffusion can actually balance the advection of the footpoints so that no strong differential rotation along the field lines is present.

Thanks to the current circuits shown in Fig. 6, we can also better understand how the toroidal field can act to collimate the outflow against the push of the centrifugal force of the rotating jet. It is in fact usually assumed that the self-generated toroidal field can automatically ensure the collimation of the outflow through the so-called "hoop stress" due to the magnetic tension of the field and equal to $-B_{\phi}^{2} / r$. What emerges clearly from both the panels of Fig. 6 is that in the outer part of the outflow, where the currents flow out from the disk with a positive $J_{z}$ component, the Lorentz force associated with the toroidal field pushes the plasma outwards, thus uncollimating the jet. In fact, in this situation the outward directed pressure gradient of the toroidal field is stronger than the collimating tension. It must be pointed out that the shape of the electric current circuits opposing the collimation of the outer layers of the outflow strongly depends on the outer boundary conditions on $B_{\phi}$, as already discussed in Sect. 2.4. On the other hand, this behavior is not just a numerical artifact, because the current circuits of our simulations have the typical butterfly-like shape of analytical models of disk-winds (see Fig. 13 in Ferreira 1997). The outflow starts to be collimated where the circuit closes back with a negative $J_{z}$ component so that the "hoop stress" exceeds the pressure gradient. As the poloidal velocity vectors in Fig. 6 show, only the inner part of the outflow in our solutions is almost cylindrically collimated inside the computational domain.

\section{Accretion rates and ejection efficiency}

After having analyzed the acceleration and collimation mechanisms, we now take into account the accretion and outflow rates that characterize our solutions. In Fig. 8 we plot for all the four simulations in which we suppressed the Ohmic heating, the accretion rates $\dot{M}_{\mathrm{a}}$ at $t=400$, defined as

$\dot{M}_{\mathrm{a}}=-2 \pi r \int_{-1.6 H}^{1.6 H} \rho u_{\mathrm{r}} \mathrm{d} z$

as a function of the cylindrical radius $r$, where $H(r)$ is the thermal height scale of the disk defined as $H=\left.\left(c_{\mathrm{s}} / \Omega_{\mathrm{K}}\right)\right|_{z=0}$. This integral is calculated up to $1.6 H$, where the magnetic diffusivity defined by Eq. (13) becomes negligible and the plasma, now in an ideal MHD regime, should flow out from the disk almost parallel to the poloidal magnetic field. It is possible to see that, for 


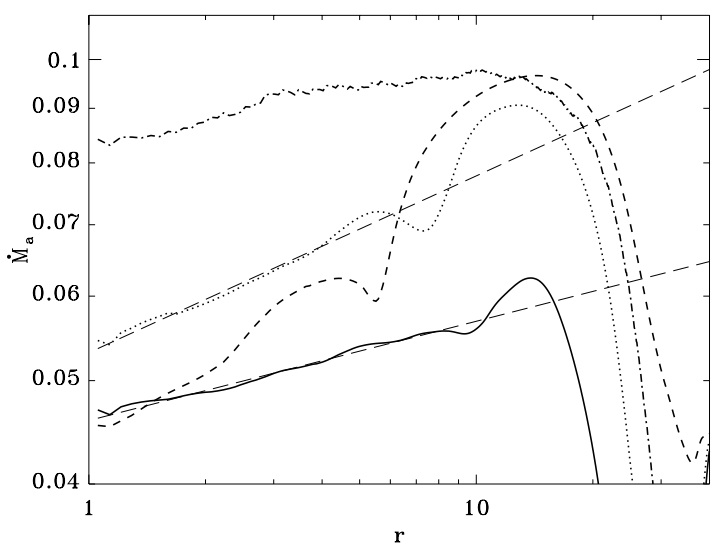

Fig. 8. Radial dependence of the accretion rates at $t=400$. The plots refer to the cases with the Ohmic heating suppressed ( $f=1)$ : $\left(\alpha_{\mathrm{m}}=1\right.$, $\chi_{\mathrm{m}}=3$ solid line $),\left(\alpha_{\mathrm{m}}=1, \chi_{\mathrm{m}}=1\right.$ dotted line $),\left(\alpha_{\mathrm{m}}=0.1, \chi_{\mathrm{m}}=1\right.$ dashed line), and $\left(\alpha_{\mathrm{m}}=0.1, \chi_{\mathrm{m}}=1\right.$, low resolution dot-dashed line line). For the two high-diffusivity cases a power-law approximation (see text) is also plotted (long-dashed line).

all the curves shown, the accretion rate has a sudden decrease for $r \gtrsim 10$, due to the fact that the outflow is efficiently extracting mass and angular momentum only for $r<10$, which is therefore identified as the "launching region". As already pointed out in Sect. 3 the outer part of the disk has still not reached a dynamically relevant time scale at the end of our simulations and therefore had no possibility of fully developing an outflow. Moreover, the flow from the inner radii of the accretion disk becomes superfast-magnetosonic inside the computational domain, and therefore the "launching region" will not be affected by the outer boundary conditions. We can therefore define a control volume delimiting this part of the disk by an inner cylinder of radius $r_{\mathrm{i}}=1$ and height $-1.6 H\left(r_{\mathrm{i}}\right)<z<1.6 H\left(r_{\mathrm{i}}\right)$ and by an outer cylinder of radius $r_{\mathrm{e}}=10$ and height $-1.6 H\left(r_{\mathrm{e}}\right)<z<1.6 H\left(r_{\mathrm{e}}\right)$. These surfaces are indicated as $\boldsymbol{S}_{\mathrm{i}}$ and $\boldsymbol{S}_{\mathrm{e}}$ respectively. Above the disk the control volume is closed at the surface determined by the height scale $1.6 H(r)$ between $r_{\mathrm{i}}=1$ and $r_{\mathrm{e}}=10$. This surface is indicated as $\boldsymbol{S}_{\mathrm{s}}$.

The four cases shown in Fig. 8 are characterized by a different outer accretion rate (calculated through $S_{\mathrm{e}}$, i.e. at $r_{\mathrm{e}}=10$ ). When the poloidal resistivity parameter $\alpha_{\mathrm{m}}$ or the anisotropy coefficient $\chi_{\mathrm{m}}$ are increased, the outer accretion rate, indicated as $\dot{M}_{\text {ae }}$, becomes smaller. It is evident also that in the cases characterized by $\alpha_{\mathrm{m}}=0.1$ the accretion rate is at least one order of magnitude higher than the initial one (see Table 1), which was determined to balance the advection and the diffusion of poloidal field lines inside the disk. It is therefore clear that the advection of the field should dominate in these cases, thus explaining the peculiar dynamical effects of the $\alpha_{\mathrm{m}}=0.1$ simulations shown in the previous section. Despite this high accretion rate, we recall that the low resistivity case at low resolution does not show these features, thus supporting the idea that the strong advection is balanced by a high numerical diffusion.

Besides being characterized by a different $\dot{M}_{\mathrm{ae}}$, the solutions also show a different slope of the accretion rate inside the "launching region". This slope is clearly linked to the amount of mass that is extracted from the disk and goes into the outflow. By defining a simplified power-law radial behavior for the accretion rate (Ferreira \& Pelletier 1995)

$\dot{M}_{\mathrm{a}}(r)=\dot{M}_{\mathrm{ae}}\left(\frac{r}{r_{\mathrm{e}}}\right)^{\xi}$

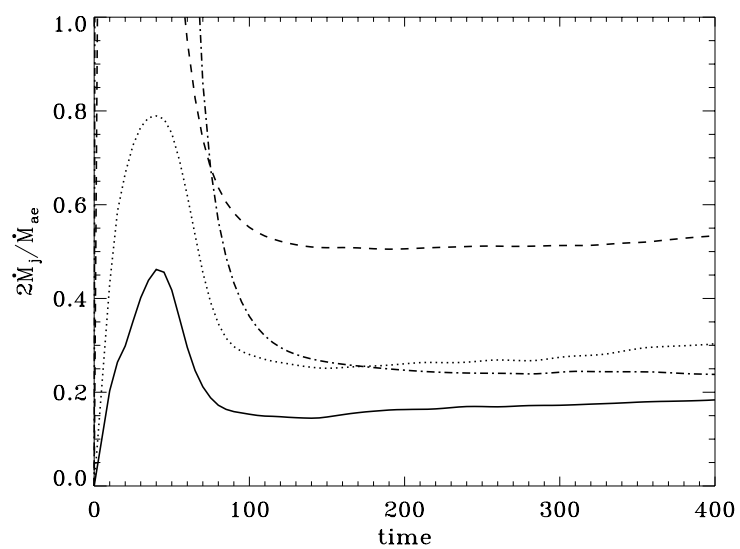

Fig. 9. Temporal evolution of the ejection efficiency. The plots refer to the cases $\left(\alpha_{\mathrm{m}}=1, \chi_{\mathrm{m}}=3\right.$ solid line $),\left(\alpha_{\mathrm{m}}=1, \chi_{\mathrm{m}}=1\right.$ dotted line $)$, $\left(\alpha_{\mathrm{m}}=0.1, \chi_{\mathrm{m}}=1\right.$ dashed line $)$, and $\left(\alpha_{\mathrm{m}}=0.1, \chi_{\mathrm{m}}=1\right.$, low resolution dot-dashed line line).

we can find, when imposing mass conservation inside the accretion disk, a simple relation between the ejection efficiency and the ejection parameter $\xi$ :

$$
\frac{2 \dot{M}_{\mathrm{j}}}{\dot{M}_{\mathrm{ae}}}=1-\left(\frac{r_{\mathrm{i}}}{r_{\mathrm{e}}}\right)^{\xi}
$$

Here the ejection efficiency $2 \dot{M}_{\mathrm{j}} / \dot{M}_{\mathrm{ae}}$ is defined as the ratio of the mass outflow from the disk surface $S_{\mathrm{s}}$ and the outer accretion rate calculated at $r_{\mathrm{e}}=10$. The factor 2 takes the two bipolar jets into account. It is easy to see that the higher the ejection efficiency, the higher the ejection parameter $\xi$ and the steeper the slope of the accretion rate. In Fig. 9 we plot the ejection efficiency $2 \dot{M}_{\mathrm{j}} / \dot{M}_{\mathrm{ae}}$ as a function of time for the four simulations taken into account in this section; and it is possible to see that after an initial transient, this efficiency reaches an almost constant value, suggesting the attainment of a stationary final state. On the other hand, the solutions reach different efficiency values, increasing from $20 \%$ for the $\left(\alpha_{\mathrm{m}}=1, \chi_{\mathrm{m}}=3\right)$ case to $55 \%$ for the $\left(\alpha_{\mathrm{m}}=0.1, \chi_{\mathrm{m}}=1\right)$ simulation. This result is consistent with the plot in Fig. 4, where we show that the forces acting vertically inside the disk change sign at lower height scales in less diffusive cases. Since the disks become denser towards the midplane, this also determines a higher outflow rate. As usual the low resistivity case at low resolution shows an anomalous behavior with an ejection efficiency typical of the $\alpha_{\mathrm{m}}=1$ cases. Moreover, the direct correlation is clear between the ejection rate and the ratio $\left|B_{\phi}\right| / B_{\mathrm{p}}$ as plotted in Fig. 5. This means that the main mechanism accelerating the outflow depends strongly on the outflow rate. The less mass-loaded jets are efficiently centrifugally accelerated, while in the more loaded jets the higher inertia strongly bends the magnetic field in the azimuthal direction right above the disk surface. As stated by Eq. (30), the outflow is therefore accelerated mainly by the pressure gradients of the toroidal field (see also Anderson et al. 2004).

Consistently the solutions characterized by a higher ejection efficiency show a steeper slope for the accretion rate as shown by Eq. (31). As an example, in Fig. 8 we plotted the power-laws calculated solving Eq. (31) for $\xi$ for the cases $\left(\alpha_{\mathrm{m}}=1, \chi_{\mathrm{m}}=3\right)$ and $\left(\alpha_{\mathrm{m}}=1, \chi_{\mathrm{m}}=1\right)$. The ejection parameters $\xi$ calculated for these two cases are $\xi=0.09$ and $\xi=0.17$, respectively. 

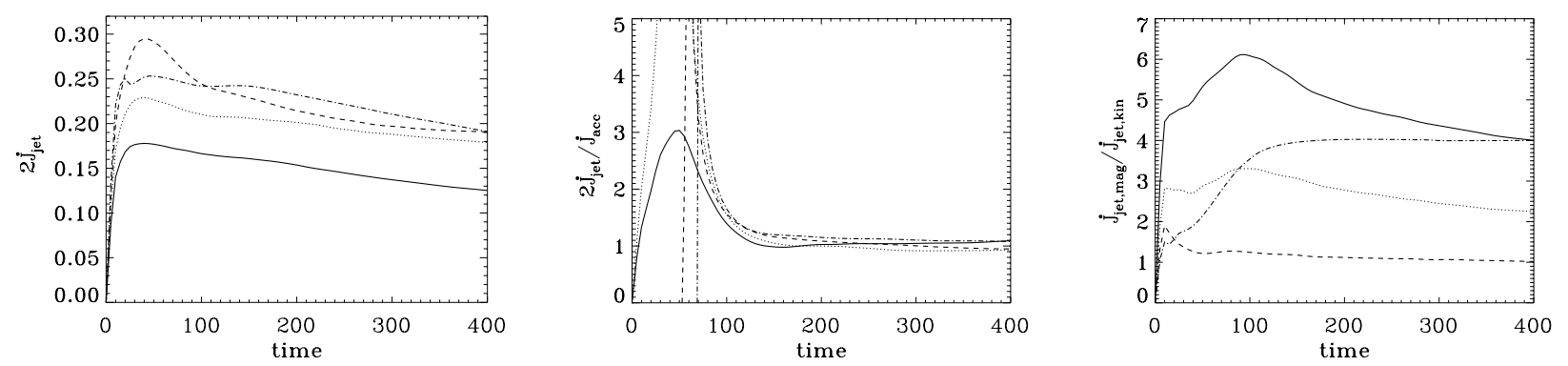

Fig. 10. Temporal evolution of the jet torque $2 \dot{J}_{\text {jet }}$ (left panel), of the ratio between the jet and accretion torque $2 \dot{J}_{\text {jet }} / \dot{J}_{\text {acc }}($ central panel), and of the ratio between the jet magnetic and mechanical torque $\dot{J}_{\text {jet,mag }} / \dot{J}_{\text {jet,kin }}$ (right panel). The plots refer to the cases $\left(\alpha_{\mathrm{m}}=1, \chi_{\mathrm{m}}=3\right.$ solid line $),\left(\alpha_{\mathrm{m}}=1\right.$, $\chi_{\mathrm{m}}=1$ dotted line $), \alpha_{\mathrm{m}}=0.1, \chi_{\mathrm{m}}=1$ dashed line $)$, and $\left(\alpha_{\mathrm{m}}=0.1, \chi_{\mathrm{m}}=1\right.$, low resolution dot-dashed line line $)$.

\section{Angular momentum transport}

It is commonly accepted that disk winds are a viable mechanism for extracting angular momentum from accretion disks without resorting to viscous torques to allow accretion. In this section we show how the bipolar jets can extract angular momentum from the underlying disk and through which channel.

The angular momentum transport is regulated by the angular momentum flux through the surface delimiting the control volume defined in Sect. 4. We can therefore define an accretion torque $\dot{J}_{\text {acc }}=\dot{J}_{\text {acc,kin }}+\dot{J}_{\text {acc, mag }}$ defined by the sum of the two terms

$\dot{J}_{\text {acc,kin }}=\int_{\boldsymbol{S}_{\mathrm{i}}} r_{\mathrm{i}} \rho u_{\phi} \boldsymbol{u} \cdot \mathrm{d} \boldsymbol{S}-\int_{\boldsymbol{S}_{\mathrm{e}}} r_{\mathrm{e}} \rho u_{\phi} \boldsymbol{u} \cdot \mathrm{d} \boldsymbol{S}$

and

$\dot{J}_{\text {acc,mag }}=\int_{S_{\mathrm{i}}} r_{\mathrm{i}} B_{\phi} \boldsymbol{B} \cdot \mathrm{d} \boldsymbol{S}-\int_{S_{\mathrm{e}}} r_{\mathrm{e}} B_{\phi} \boldsymbol{B} \cdot \mathrm{d} \boldsymbol{S}$.

For the accretion torque we use a positive sign if it increases the angular momentum contained inside the control volume and a negative sign if it extracts it. The term $\dot{J}_{\text {acc,kin }}$ defines the flux of angular momentum inside the control volume due to the accretion motion, while $\dot{J}_{\text {acc,mag }}$ is the magnetic torque that can transport angular momentum along the radial direction inside the disk. This magnetic torque represents a small contribution to $\dot{J}_{\text {acc }}$ : in all of our simulations the ratio $\dot{J}_{\text {acc,mag }} / \dot{J}_{\text {acc,kin }}$ is always negligible, around $-10 \%$. A small fraction of the disk angular momentum is also extracted by the magnetic torque radially, thus helping accretion. On the other hand, the main contribution to $\dot{J}_{\text {acc,kin }}$ is given by the integral on the outer radius, where both the accretion rate and the specific angular momentum of the disk are higher. Assuming a Keplerian rotation, a reasonable approximation for $\dot{J}_{\text {acc }}$ is therefore given by (see also Eq. (28)):

$\dot{J}_{\mathrm{acc}} \sim \dot{M}_{\mathrm{ae}} \sqrt{G M r_{\mathrm{e}}}$.

In the same way, we can define the torque exerted by the outflow on the disk $\dot{J}_{\text {jet }}=\dot{J}_{\text {jet,kin }}+\dot{J}_{\text {jet,mag }}$ as the sum of the flux of angular momentum $\dot{J}_{\text {jet,kin }}$ and of the magnetic torque $\dot{J}_{\text {jet,mag }}$ at the disk surface:

$\dot{J}_{\text {jet,kin }}=\int_{\boldsymbol{S}_{\mathrm{s}}} r \rho u_{\phi} \boldsymbol{u} \cdot \mathrm{d} \boldsymbol{S}$

and

$\dot{J}_{\text {jet,mag }}=\int_{S_{\mathrm{s}}} r B_{\phi} \boldsymbol{B} \cdot \mathrm{d} \boldsymbol{S}$.

For the jet torque we use a positive sign if it extracts angular momentum from the disk.
The accretion and the jet torque should be equal in a steady situation: $2 \dot{J}_{\text {jet }}=\dot{J}_{\text {acc }}$. Using the approximation made in Eq. (34), the relation

$\dot{M}_{\mathrm{ae}} \sqrt{G M r_{\mathrm{e}}} \sim 2 \dot{J}_{\text {jet }}$

shows clearly that the accretion rate at the outer radius of the launching region $r_{\mathrm{e}}$ is mainly controlled by the torque exerted by the outflow on the accretion disk. In the left panel of Fig. 10, we plot the temporal evolution of the total torque $2 \dot{J}_{\text {jet }}$ exerted by the bipolar jets of the four simulations performed without Joule heating: the correlation is clear between the outer accretion rate shown in Fig. 8 and the jet torque plotted here, as stated by Eq. (37). The high accretion rate of the $\alpha_{\mathrm{m}}=0.1$ cases, which determines the peculiar behavior of these solutions, is determined by the high torque exerted by the jet, at least ten times higher than the torque needed to maintain the initial rate (see Table 1). The curves plotted in the left panel of Fig. 10 show a slow decrease in time, thus suggesting that our solutions did not reach a final steady state. On the other hand, the ratio $2 \dot{J}_{\text {jet }} / \dot{J}_{\text {acc }}$, plotted in the central panel of Fig. 10 , stays approximatively constant in time and equal to one, after an initial transient, which shows that the accretion rate of the disk reacts quickly to the slowly varying jet torque, thus suggesting that our simulations are evolving through a series of quasi-stationary states.

Even if the outflows of the different simulations are exerting almost the same torque (left panel, Fig. 10), being a factor two smaller just in the case characterized by a high and anisotropic magnetic diffusivity, the ratio between the magnetic $\dot{J}_{\text {jet,mag }}$ and the mechanical torque $\dot{J}_{\text {jet,kin }}$ shows noticeable differences (right panel in Fig. 10). The ratio goes from a value around unity for the less dissipative case $\left(\alpha_{\mathrm{m}}=0.1\right.$, $\left.\chi_{\mathrm{m}}=1\right)$ up to a value $\gtrsim 4$ for the $\left(\alpha_{\mathrm{m}}=1, \chi_{\mathrm{m}}=3\right)$ simulation. Once again, we point out that the low resistivity simulation at low resolution behaves like a higher resistivity case, showing $\dot{J}_{\text {jet,mag }} / \dot{J}_{\text {jet,kin }} \sim 4$. This quantity has an easy interpretation: the angular momentum extracted from the disk is in fact stored initially in the toroidal magnetic field and is then transferred starting from the disk surface to the outflowing plasma, which is therefore centrifugally accelerated. The ratio $\dot{J}_{\text {jet,mag }} / \dot{J}_{\text {jet,kin }}$ is therefore a measure of the efficiency of the magneto-centrifugal mechanism. The higher this ratio, the more specific angular momentum is available at the disk surface, centrifugally accelerating the plasma to higher poloidal terminal speeds (see Sect. 6).

\section{Energy budget of the disk-jet system}

In this section we finally study the energetics of the disk-jet system. To study the energy balance of our simulations we consider 

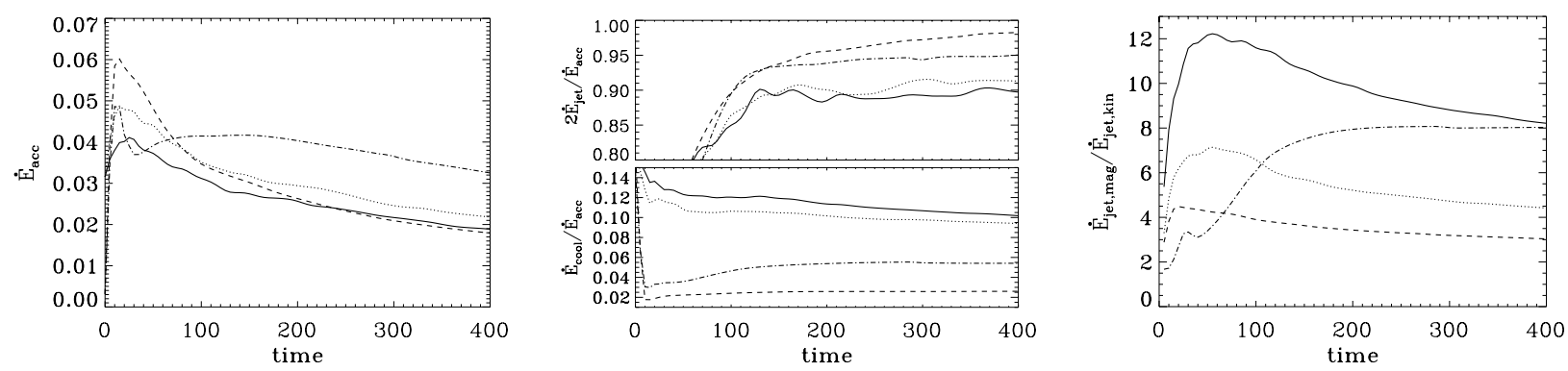

Fig. 11. Temporal evolution of the accretion power $\dot{E}_{\text {acc }}$ (left panel); the ratio between the jet and accretion power $2 \dot{E}_{\text {jet }} / \dot{E}_{\text {acc }}$ and the ratio between the radiated and accretion power $2 \dot{E}_{\text {jet }} / \dot{E}_{\text {acc }}$ (central panel); the ratio between the jet magnetic and kinetic power $\dot{E}_{\text {jet,mag }} / \dot{E}_{\text {jet,kin }}($ right panel). The plots refer to the cases $\left(\alpha_{\mathrm{m}}=1, \chi_{\mathrm{m}}=3\right.$ solid line $),\left(\alpha_{\mathrm{m}}=1, \chi_{\mathrm{m}}=1\right.$ dotted line $),\left(\alpha_{\mathrm{m}}=0.1, \chi_{\mathrm{m}}=1\right.$ dashed line $)$, and $\left(\alpha_{\mathrm{m}}=0.1, \chi_{\mathrm{m}}=1\right.$, low resolution dot-dashed line line).

Table 2. Values of the different contributions to the total accretion power $\dot{E}_{\text {acc }}$ calculated at $t=400$.

\begin{tabular}{cccc}
\hline \hline $\begin{array}{c}\text { Simulation } \\
\left(\alpha_{\mathrm{m}}, \chi_{\mathrm{m}}\right)\end{array}$ & $\dot{E}_{\text {acc,mec }} / \dot{E}_{\text {acc }}$ & $\dot{E}_{\text {acc,mag }} / \dot{E}_{\text {acc }}$ & $\dot{E}_{\text {acc,thm }} / \dot{E}_{\text {acc }}$ \\
\hline$(0.1,1)$ & 0.97 & 0.14 & -0.11 \\
$(0.1,1)$ low $r$. & 1.29 & 0.09 & -0.38 \\
$(1,1)$ & 0.97 & 0.10 & -0.07 \\
$(1,3)$ & 0.99 & 0.05 & -0.04 \\
\hline
\end{tabular}

the energy fluxes through the surfaces of the control volume defined in Sect. 4 . We then define an accretion power $\dot{E}_{\text {acc }}=$

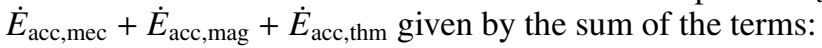

$$
\begin{aligned}
& \dot{E}_{\mathrm{acc}, \mathrm{mec}}=\int_{\boldsymbol{S}_{\mathrm{i}}}\left(\frac{u^{2}}{2}+\Phi_{\mathrm{g}}\right) \rho \boldsymbol{u} \cdot \mathrm{d} \boldsymbol{S}-\int_{\boldsymbol{S}_{\mathrm{e}}}\left(\frac{u^{2}}{2}+\Phi_{\mathrm{g}}\right) \rho \boldsymbol{u} \cdot \mathrm{d} \boldsymbol{S} \\
& \dot{E}_{\mathrm{acc}, \mathrm{mag}}=\int_{\boldsymbol{S}_{\mathrm{i}}} \boldsymbol{E} \times \boldsymbol{B} \cdot \mathrm{d} \boldsymbol{S}-\int_{\boldsymbol{S}_{\mathrm{e}}} \boldsymbol{E} \times \boldsymbol{B} \cdot \mathrm{d} \boldsymbol{S} \\
& \dot{E}_{\mathrm{acc}, \mathrm{thm}}=\int_{\boldsymbol{S}_{\mathrm{i}}} \frac{\gamma}{\gamma-1} P \boldsymbol{u} \cdot \mathrm{d} \boldsymbol{S}-\int_{\boldsymbol{S}_{\mathrm{e}}} \frac{\gamma}{\gamma-1} P \boldsymbol{u} \cdot \mathrm{d} \boldsymbol{S}
\end{aligned}
$$

where $\dot{E}_{\text {acc,mec }}, \dot{E}_{\text {acc,mag }}$ and $\dot{E}_{\text {acc,thm }}$ represent the mechanical (kinetic + gravitational), Poynting, and enthalpy flux through the cylindrical surfaces at the external and internal radius of the launching region. For the accretion power we use a positive sign if it increases the energy contained inside the control volume. In the left panel of Fig. 11 we plot the temporal evolution of the total accretion power, while in Table 2 we show the different contribution to $\dot{E}_{\mathrm{acc}}$ at $t=400$ for the cases considered in this section. In the table the simulations are characterized by their diffusivity parameters $\alpha_{\mathrm{m}}$ and $\chi_{\mathrm{m}}$ ). We can see that the dominant term is always the mechanical power liberated in the accretion. The Poynting flux slightly increases the total energy contained in the control volume, while the enthalpy flux always acts to decrease the accretion power, advecting the thermal energy at the inner radius. It is important to notice that the simulation with a low magnetic diffusivity performed at a lower resolution (second line) shows a higher enthalpy flux due to the higher numerical dissipation. Neglecting the enthalpy and the magnetic contribution and assuming a Keplerian rotation, the energy liberated by accretion can be safely approximated by (see also Eq. (28)):

$\dot{E}_{\mathrm{acc}} \sim \dot{M}_{\mathrm{ai}} \frac{G M}{2 r_{\mathrm{i}}}-\dot{M}_{\mathrm{ae}} \frac{G M}{2 r_{\mathrm{e}}}$.

This approximation shows that the liberated power is mainly determined by the inner accretion rate, and the values of $\dot{E}_{\text {acc }}$ of
Table 3. Values of the different contributions to the total jet power $\dot{E}_{\text {jet }}$ calculated at $t=400$.

\begin{tabular}{ccccc}
\hline \hline $\begin{array}{c}\text { Simulation } \\
\left(\alpha_{\mathrm{m}}, \chi_{\mathrm{m}}\right)\end{array}$ & $\dot{E}_{\text {jet,kin }} / \dot{E}_{\mathrm{jet}}$ & $\dot{E}_{\text {jet,grv }} / \dot{E}_{\text {jet }}$ & $\dot{E}_{\text {jet,mag }} / \dot{E}_{\text {jet }}$ & $\dot{E}_{\text {jet,thm }} / \dot{E}_{\text {jet }}$ \\
\hline$(0.1,1)$ & 0.42 & -0.73 & 1.28 & 0.03 \\
$(0.1,1)$ low $r$. & 0.13 & -0.24 & 1.05 & 0.06 \\
$(1,1)$ & 0.25 & -0.49 & 1.22 & 0.02 \\
$(1,3)$ & 0.13 & -0.26 & 1.12 & 0.01 \\
\hline
\end{tabular}

our simulations at $t=400$ (left panel of Fig. 11) are clearly correlated with the inner accretion rates visible in Fig. 8.

To characterize the energy extracted by the outflow, we define a jet power $\dot{E}_{\text {jet }}=\dot{E}_{\text {jet,kin }}+\dot{E}_{\text {jet,grv }}+\dot{E}_{\text {jet,mag }}+\dot{E}_{\text {jet,thm given }}$ by the sum of the fluxes of kinetic, gravitational, magnetic, and thermal energy at the disk surface $\boldsymbol{S}_{\mathrm{s}}$ :

$$
\begin{aligned}
& \dot{E}_{\text {jet,kin }}=\int_{\boldsymbol{S}_{\mathrm{s}}} \frac{u^{2}}{2} \rho \boldsymbol{u} \cdot \mathrm{d} \boldsymbol{S} \\
& \dot{E}_{\text {jet,grv }}=\int_{\boldsymbol{S}_{\mathrm{s}}} \Phi_{\mathrm{g}} \rho \boldsymbol{u} \cdot \mathrm{d} \boldsymbol{S} \\
& \dot{E}_{\text {jet,mag }}=\int_{\boldsymbol{S}_{\mathrm{s}}} \boldsymbol{E} \times \boldsymbol{B} \cdot \mathrm{d} \boldsymbol{S} \\
& \dot{E}_{\text {jet,thm }}=\int_{\boldsymbol{S}_{\mathrm{s}}} \frac{\gamma}{\gamma-1} P \boldsymbol{u} \cdot \mathrm{d} \boldsymbol{S} .
\end{aligned}
$$

For the jet power we use a positive sign if it extracts energy from the control volume.

Since in this section we are considering cases characterized by $f=1$, we can also define the radiated power $\dot{E}_{\text {cool }}$ as

$\dot{E}_{\text {cool }}=\int_{V_{\mathrm{c}}} \Lambda_{\text {cool }} \mathrm{d} V=\int_{V_{\mathrm{c}}} \overline{\overline{\boldsymbol{\eta}}} \boldsymbol{J} \cdot \boldsymbol{J} \mathrm{d} V$

where $V_{\mathrm{c}}$ is the control volume. In a steady situation the energy fluxes should balance to give $2 \dot{E}_{\text {jet }}=\dot{E}_{\text {acc }}-\dot{E}_{\text {cool }}$. In the central panel of Fig. 11 we plot the time evolution of the ratios $2 \dot{E}_{\text {jet }} / \dot{E}_{\text {acc }}$ and $\dot{E}_{\text {cool }} / \dot{E}_{\text {acc }}$. We can see that most of the accretion power is liberated in the jet, increasing from $\sim 90 \%$ for the more dissipative case $\left(\alpha_{\mathrm{m}}=1, \chi_{\mathrm{m}}=3\right)$ up to $\sim 98 \%$ for the less dissipative one $\left(\alpha_{\mathrm{m}}=0.1, \chi_{\mathrm{m}}=1\right)$. Correspondingly the radiated power decreases from $\sim 10 \%$ down to $\sim 2 \%$. The two contibutions sum up to approximately one, suggesting a quasi-stationary situation.

As noticed for the jet torques, the jet powers also do not show a huge difference between the simulations. On the other hand, the different contributions to the total jet power more clearly depend on the simulation parameters. In Table 3 we see that the 


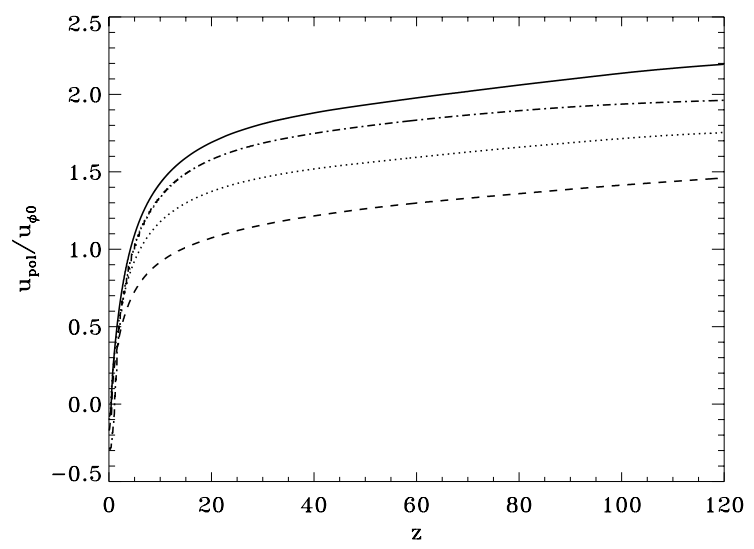

Fig. 12. Average poloidal speed $\mathrm{u}_{\mathrm{pol}}$ along the jets calculated at $t=400$, normalized to the value of the toroidal (Keplerian) speed $u_{\phi 0}$ at the base of each streamline. These curves were obtained by averaging the ratio $u_{\mathrm{pol}} / u_{\phi 0}$ on all the streamlines flowing out from the upper boundary of the computational box. The line style used refers to the same cases as in Fig. 11.

greater contribution to the jet power at the surface of the disk comes from the Poynting flux, since most of the energy is stored in the toroidal magnetic field. The enthalpy flux is always negligible, because our jets are cold, undergoing a continuous adiabatic expansion starting from the disk surface. The kinetic and the gravitational fluxes are strongly correlated with the outflow rates of the different cases, but the gravitational contribution is negative since the outflow at the disk surface is still inside the potential well of the central object. In the right panel of Fig. 11 we plot the temporal evolution of the ratio between the jet Poynting

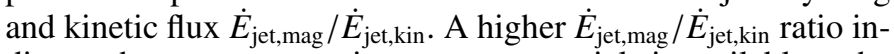
dicates that more magnetic energy per particle is available at the disk surface to accelerate the outflow. The Poynting flux available at the disk surface is then converted into kinetic energy along the outflow, and at the upper end of the computational box, the kinetic flux is dominant, as confirmed by the fact that the flow is super-fast-magnetosonic. The asymptotic speed of the outflow is therefore higher for cases characterized by a higher $\dot{E}_{\text {jet,mag }} / \dot{E}_{\text {jet,kin }}$, as confirmed by Fig. 12 where we plot an average value of the poloidal speed normalized to the toroidal (Keplerian) speed at the base of each streamline. The poloidal velocity measured at the upper end of the computational domain is a few times the Keplerian speed at the base of the streamlines, which is consistent with the fact that different classes of astrophysical jets show velocities on the same order as the escape velocity from the central object (Livio 1999).

\section{A quasi-steady solution}

Here we try to characterize one of the simulations performed as a steady solution. We have already pointed out that none of our solutions are perfectly steady, because the low diffusivity case shows a continuously evolving magnetic structure, while all the simulations show a slowly evolving jet torque (left panel in Fig. 10) and accretion power (left panel in Fig. 11). On the other hand, the constant values assumed by the accretion efficiency (Fig. 9), by the ratio between the jet and the accretion torque (central panel in Fig. 10), and by the ratio between the jet and the accretion power (central panel in Fig. 11) indicate that our solutions, at least those with $\alpha_{\mathrm{m}}=1$, are slowly evolving through a series of quasi-stationary states. In this section we analyze the simulation characterized by $\alpha_{\mathrm{m}}=1, \chi_{\mathrm{m}}=3$, and

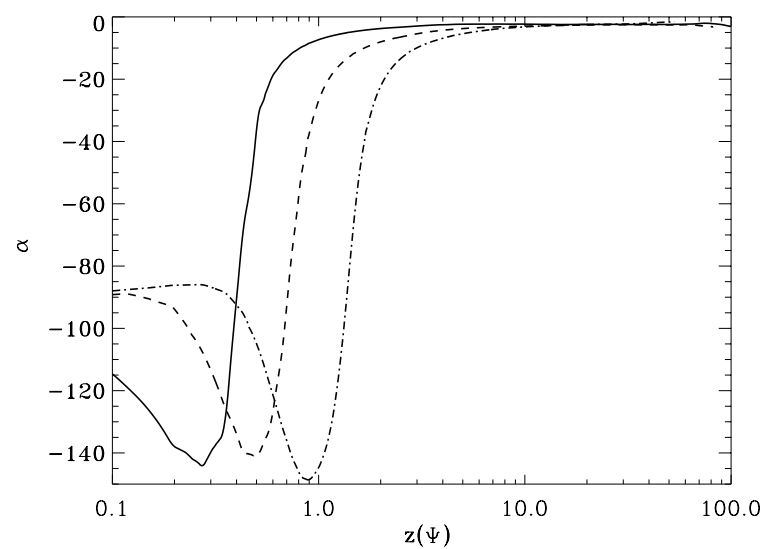

Fig. 13. Angle between poloidal speed and poloidal magnetic field for the case characterized by $\alpha_{\mathrm{m}}=1, \chi_{\mathrm{m}}=3$ and with the Ohmic heating suppressed. This quantity is calculated at $t=400$ along three sample field lines whose footpoints are located at $r_{\mathrm{F}}=2$ (solid line), 4 (dashed line), and 8 (dot-dashed line).

$f=1$. Besides showing some features of a stationary solution, its parameters $\alpha_{\mathrm{m}}, \chi_{\mathrm{m}}, \mu$, and $\epsilon$ are typical of the cold self-similar steady solutions found by Casse \& Ferreira (2000a), allowing a direct comparison between steady and time-dependent solutions.

We recall a few relations that are valid for a steady axisymmetric solution of the ideal MHD equations. The poloidal speed $\boldsymbol{u}_{\mathrm{p}}$ is related to the poloidal magnetic field $\boldsymbol{B}_{\mathrm{p}}$

$\boldsymbol{u}_{\mathrm{p}}=\frac{K(\Psi)}{\rho} \boldsymbol{B}_{\mathrm{p}}$,

where $K(\Psi)$ is a constant along every field line marked by the flux function $\Psi$, and it represents the ratio between the mass flux and the magnetic flux. This relation clearly states that the poloidal speed and magnetic field are parallel in a steady situation. The toroidal speed $u_{\phi}$ is related to the rotation rate of the magnetic surface $\Omega(\Psi)$ by

$u_{\phi}=\Omega(\Psi) r+\frac{K(\Psi)}{\rho} B_{\phi}$

The total specific angular momentum $l(\Psi)$, which is constant along each field line, is given by

$l(\Psi)=r u_{\phi}-\frac{r B_{\phi}}{K(\Psi)}=\Omega r_{\mathrm{A}}^{2}$

where $r_{\mathrm{A}}$ is the Alfvén radius. Each field line is therefore characterized by the non-dimensional constants $k$, or mass loading parameter, and $\lambda$, which gives a measure of the magnetic lever $\operatorname{arm} r_{\mathrm{A}} / r_{\mathrm{F}}$ :

$k=K(\Psi) \frac{r_{\mathrm{F}} \Omega}{B_{0}}$

$\lambda=\frac{l(\Psi)}{\Omega r_{\mathrm{F}}}=\left(\frac{r_{\mathrm{A}}}{r_{\mathrm{F}}}\right)^{2}$,

where $r_{\mathrm{F}}$ is the cylindrical radius of the footpoint of the field line and $B_{0}$ is the value of the magnetic field at $r_{\mathrm{F}}$.

We therefore tried to characterize our simulation computing these steady invariants along three sample field lines whose footpoints are located at $r_{\mathrm{F}}=2,4$, and 8. In Fig. 13 the angle is plotted between the poloidal field and the poloidal speed. While inside the disk, where the magnetic resistivity is effective, the accreting plasma flows perpendicular to the field lines, in the outflow, where the ideal MHD holds, the matter flows along the 


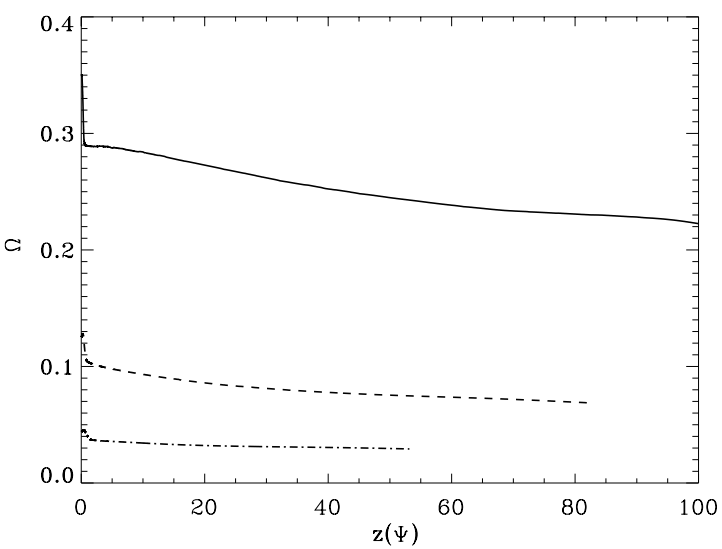

Fig. 14. Rotation rate of the magnetic field calculated along the fieldlines, whose footpoints are located at $r_{\mathrm{F}}=2$ (solid line), 4 (dashed line), and 8 (dot-dashed line).

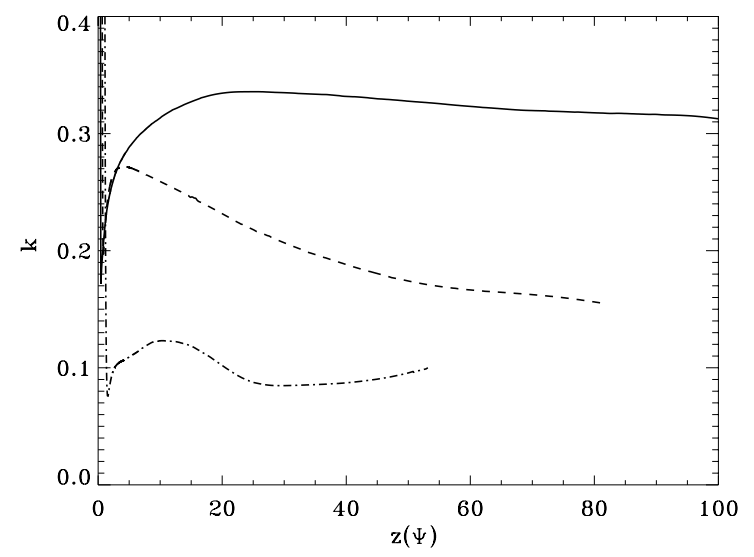

Fig. 15. Mass-loading parameter calculated along the fieldlines whose footpoints are located at $r_{\mathrm{F}}=2$ (solid line), 4 (dashed line), and 8 (dotdashed line).

lines. In Fig. 14 the $\Omega$ invariant is plotted: the rotation rate of the field lines, defined by a reference frame in which the electric field is zero, is close to the Keplerian rate, since the field lines are anchored in the accretion disk and corotate with it. Moreover, it is possible to see that $\Omega$ is slightly higher at the base of the outflow. As in the low resistivity case but without noticeable consequences, the footpoint of the field line is advected towards the central part of the disk, rotating a little bit faster than its outer end.

The mass loading parameter $k$ is plotted in Fig. 15, and the $\lambda$ invariant is shown in Fig. 16. The two quantities show a much more constant behavior along the inner field line, indicating that the central part of the outflow has reached a more stationary state, while the outer part of it is still slowly evolving. A clear trend emerges in these plots. The mass-loading decreases going from the inner to the outer parts of the outflow while the magnetic lever arm increases. The relation between the $k$ and $\lambda$ parameters is shown in Fig. 17, where we plotted the values of these quantities calculated at the Alfvén point on different fieldlines anchored in the disk. The higher values of $k$ are found on the inner $(r \sim 1)$ fieldlines, whereas the lower ones are in the outer part $(r \sim 10)$ of the launching region. The following relation is also plotted for comparison:

$\lambda=\frac{3}{2}\left(1+k^{-2 / 3}\right)$

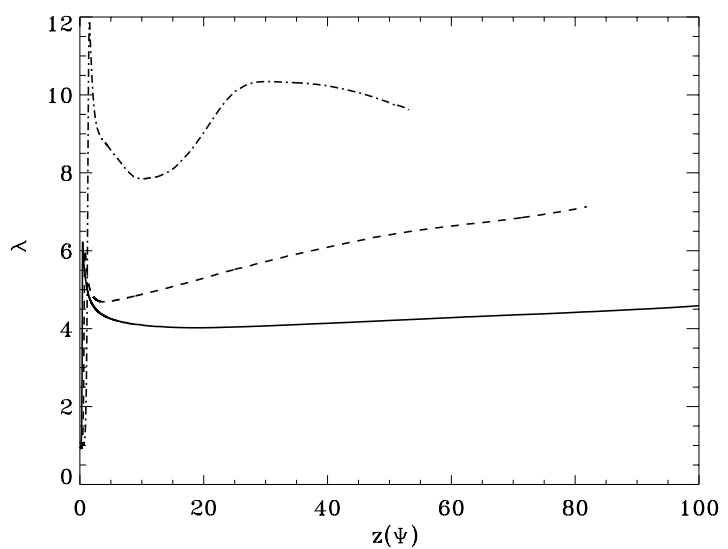

Fig. 16. Magnetic lever arm parameter calculated along the fieldlines whose footpoints are located at $r_{\mathrm{F}}=2$ (solid line), 4 (dashed line), and 8 (dot-dashed line).

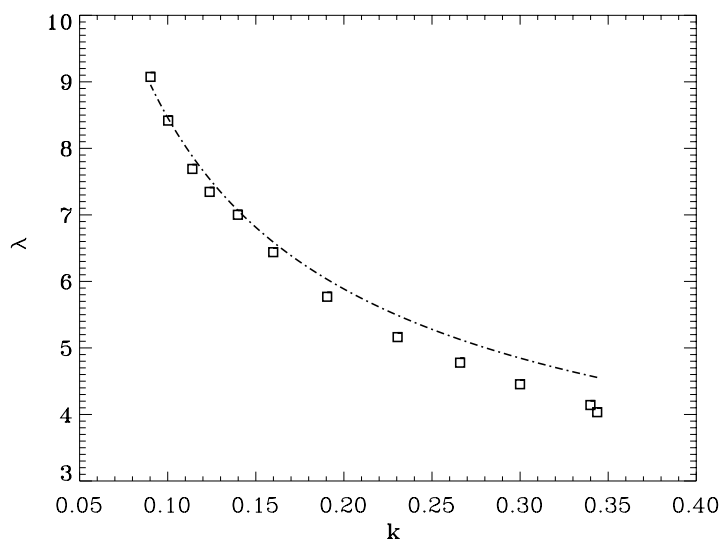

Fig. 17. $k-\lambda$ relation (squares). The values are calculated at the Alfven point along different fieldlines anchored in the launching region of the disk $(1<r<10)$. For comparison the relation Eq. (52) found by Weber \& Davis (1967) for a radial wind geometry is also plotted (dot-dashed line).

found by Weber \& Davis (1967) for a radial wind geometry. The rough behavior $\lambda \propto k^{-2 / 3}$ is also found by Ouyed \& Pudritz (1997) and Anderson et al. (2004).

We point out that the following relation is valid in cold (adiabatic) steady solutions (see e.g. Blandford \& Payne 1982) along each field line:

$\frac{\dot{E}_{\text {jet,mag }}}{\dot{E}_{\text {jet,kin }}}=2 \frac{\dot{J}_{\text {jet,mag }}}{\dot{J}_{\text {acc,kin }}}=2(\lambda-1)$.

We plotted an averaged value over the disk surface of these two ratios in Figs. 10 and 11. The magnetic lever arm is therefore a measure of the efficiency of the magneto-centrifugal mechanism. Assuming that all the Poynting flux available at the disk surface is completely converted into poloidal kinetic energy, the following relation for the poloidal asymptotic speed of the outflow $u_{\mathrm{p}, \infty}$ holds:

$u_{\mathrm{p}, \infty}=\Omega r_{\mathrm{F}} \sqrt{2 \lambda-3}$

Therefore the poloidal speed, expressed in units of the rotation speed at the footpoint, asymptotically assumes higher values on the outer field lines.

Finally, it is interesting to compare our solution with the cold self-similar steady solutions found by Casse \& Ferreira (2000a) 
characterized by the same parameters $\alpha_{\mathrm{m}}, \chi_{\mathrm{m}}, \epsilon$, and $\mu$. The simulation presented in this section shows higher values of the mass loading $k$ and of the ejection parameter $\xi \sim 0.1$ (calculated in Sect. 4) and lower magnetic lever arms. The steady solution, characterized approximately by $k \sim 2 \times 10^{-2}, \xi \sim 10^{-2}$, and $\lambda \sim 35$, therefore has a much lower outflow rate and higher terminal speed. A possible physical explanation is given by the shape of the current circuits shown in Sect. 3.2. Ferreira (1997) has shown that high ejection efficiencies $(\xi>0.5)$ are obtained if the poloidal current enters the disk at its surface and that no steady solution crossing all the critical points is possible. Its solutions are always characterized by a lower ejection parameter $\xi<0.5$ and by a current outflowing from the disk surface. In our solutions the current returns and enters the disk in the small region $1<r<2$ near the central sink region. Due to the internal boundary itself the rotation of the outflow suddenly goes to zero at the inner edge of the jet, creating a current sheet that is forced to flow back inside the disk. This is therefore a numerical effect, and a proper treatment of the region of interaction between the disk and the central object is required to understand how the currents close in the inner region of the system. It is at least possible to speculate that the returning current increases the ejection efficiency as proposed by Ferreira (1997), which can also be confirmed by the fact that in our solution the mass loading parameter $k$ increases towards the center, where the current flows back into the disk. Another possible explanation is determined by the numerical dissipation that can increase the thermal energy and the pressure in the launching region, increasing the outflow rate as shown in Sect. 3.1 (see also Casse \& Ferreira $2000 \mathrm{~b}$ ). If, on one hand, we have shown that a low resolution yields the effects of a high disk diffusivity, on the other hand, we cannot be confident that the standard resolution used in the simulations is sufficient to accurately resolve the vertical structure of the accretion disk. It is well known how difficult it is for the type of algorithms used in this paper to handle low densities, such as in the launching region, where the disk expands a lot. This is also suggested by the behavior of the invariants $k$ and $\lambda$, e.g. on the inner field line considered, which show a slow transition to their more or less constant values, indicating that the transition between the resistive and the ideal MHD regimes happens on a larger scale than the one determined by the vertical profile of the diffusivity (Eq. (13)).

\section{Effects of Ohmic heating}

We finally make a few considerations about the two simulations performed in which all the dissipated magnetic energy is released locally inside the disk $(f=0)$, and we compare the corresponding cases in which the dissipated energy is radiated away $(f=1)$. We recall that these two simulations are characterized by $\left(\alpha_{\mathrm{m}}=0.1, \chi_{\mathrm{m}}=1\right)$ and $\left(\alpha_{\mathrm{m}}=1, \chi_{\mathrm{m}}=3\right)$.

The dissipative Ohmic term $\Lambda=\overline{\bar{\eta}} \boldsymbol{J} \cdot \boldsymbol{J}$ now acts to increase the thermal energy and the entropy of the plasma inside the disk. This has two important consequences: the energy dissipation at the midplane changes the disk thickness and modifies its structure, while an increase in the pressure gradient at the disk surface allows more matter to be loaded in the outflow.

In Fig. 18 we plot the time evolution of the ejection efficiency of the two "hot" solutions and of the two corresponding "cold" simulations. Both the "hot" simulations are characterized by a higher efficiency; moreover, the case with $\alpha_{\mathrm{m}}=1$ shows a steep increase at the end of the simulation. This is due both to a decrease in the outer accretion rate and to an increase in the outflow rate. The energy dissipation steepens the radial gradient

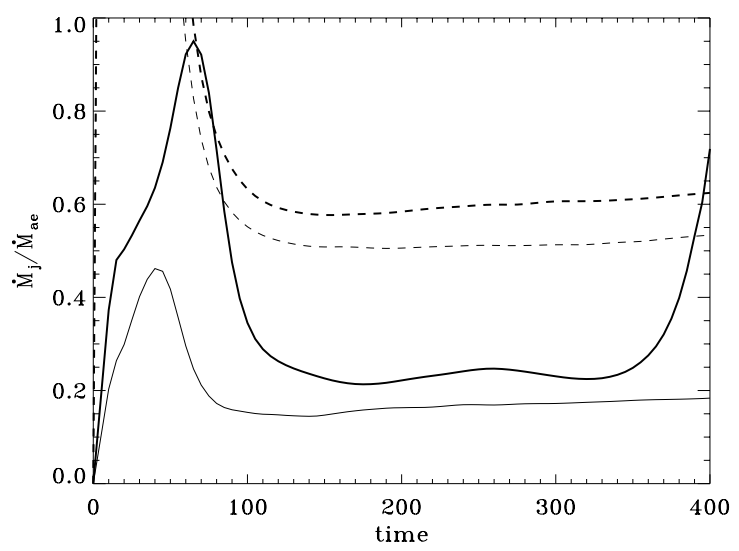

Fig. 18. Temporal evolution of the ejection efficiency for the simulations in which the magnetic energy is dissipated locally as Joule heating: $\left(\alpha_{\mathrm{m}}=1, \chi_{\mathrm{m}}=3\right.$, solid thick line $)$, and $\alpha_{\mathrm{m}}=0.1, \chi_{\mathrm{m}}=1$, dashed thick line). With a thinner line we also plot the curves that refer to the corresponding cases in which the dissipated energy is radiated.

of the thermal pressure inside the disk, thus slowing the outer accretion down. At the inner radius the gradient is so steep that the disk does not accrete anymore and all the matter is pushed by the pressure gradient into a highly unsteady outflow.

The disk's thermal energy of the "cold" cases is almost constant in time. The "hot" case with $\alpha_{\mathrm{m}}=0.1$ shows a similar behavior, with a disk thermal energy just slightly higher than the corresponding "cold" simulation. It is likely that the higher outflow rate can balance the small increase in the disk thermal energy, which is equivalent to the $2 \%$ of the accretion power that was "radiated" in the corresponding "cold" simulation (see Sect. 6). On the other hand, the disk's thermal energy of the "hot" counterpart of the $\alpha_{\mathrm{m}}=1$ simulation continuously increases, not balanced by the energy extracted by the outflow, in turn leading to the unsteady behavior shown in Fig. 18. It is important to notice that the behavior of this simulation depends a lot on the expression that we used for the magnetic diffusivity (Eq. (13)), which is proportional to the disk thermal height scale. The Ohmic heating in fact determines an increase in the disk thickness that then implies a higher magnetic diffusivity.

\section{Summary and conclusions}

Our calculations have followed the long-term evolution of an axisymmetric quasi-Keplerian magnetized disk up to the establishment of an inflow/outflow configuration. The accretion flow is driven by extraction of angular momentum of the disk by the jet, which is shown by reaching a balance between the accretion and jet torques. The magnetic torque of the jet is most efficient close to the surface of the disk extracting angular momentum from the accretion flow; it stores angular momentum in the toroidal magnetic field that then accelerates the outflowing plasma. Therefore the simulations have succeeded in demonstrating that the magnetocentrifugal mechanism originally proposed by Blandford \& Payne can launch jets, provided certain physical conditions on the magnetic resistivity and initial field configuration are satisfied.

In particular we have shown that an isotropic $\left(\chi_{\mathrm{m}}=1\right)$ resistive configuration with $\alpha_{\mathrm{m}}=0.1$, due to the stronger advection of the field compared to its diffusion, produces highly unsteady magnetic structures, as displayed in Fig. 2 and in the left panel of Fig. 7. This agrees with the stationary models of Casse \& Ferreira (2000a) according to which it is not possible to obtain 
a steady outflow with such a low $\alpha_{\mathrm{m}}$ parameter except for highly anisotropic configurations $\left(\chi_{\mathrm{m}}>10^{2}\right)$. This would agree with the results shown in Sect. 5, because a much stronger toroidal resistivity should reduce the torque exerted by the jet on the disk and thereby its accretion rate (Eq. (37)). On the other hand, we also showed how this unsteady behavior depends on the resolution assumed and therefore on the numerical dissipation: the same case $\alpha_{\mathrm{m}}=0.1$ performed with a four times lower resolution presents many of the characteristics of a solution with $\alpha_{\mathrm{m}}=1$. It can be presumed that the quasi-stationary behavior found by Casse \& Keppens $(2002,2004)$ with an isotropic $\alpha_{\mathrm{m}}=0.1$ parameter can be affected by the resolution used.

On the other hand, the cases characterized by $\alpha_{\mathrm{m}}=1$ are also not perfectly stationary, despite showing an ordered magnetic configuration favorable to a steady launching. In all the simulations performed some integrated quantities, like the jet torque (Fig. 10) or the accretion energy (Fig. 11), are still slowly evolving. Even the case characterized by an anisotropic $\left(\chi_{\mathrm{m}}=3\right)$ diffusivity, needed by the stationary models, does not show a perfectly stationary behavior. Moreover, it can be characterized by adimensional quantities like $\xi, k$ or $\lambda$ which differ a lot from the analogous ones found in the cold solutions of Casse \& Ferreira (2000a). In Sect. 7 we proposed some physical and numerical reasons for this behavior.

Nevertheless the constant value assumed in time by some quantities, like the ejection efficiency (see Fig. 9) or the ratio between the accretion power and the jet energy flux (central panel of Fig. 11) suggests that our solutions are slowly evolving through a series of quasi-stationary states. A clear trend emerges. When increasing the poloidal and/or the toroidal resistivity, the ejection efficiency decreases, from $55 \%$ in the $\left(\alpha_{\mathrm{m}}=0.1, \chi_{\mathrm{m}}=\right.$ $1)$ case to $20 \%$ for the $\left(\alpha_{\mathrm{m}}=1, \chi_{\mathrm{m}}=3\right)$ simulation. From the energetic point of view, our simulations show that more than $90 \%$ of the energy liberated in the accretion inflow is released in the jet and that less dissipative cases produce slightly more powerful jets. Correspondingly, the radiated power that must take care of the Joule heating dissipation is less than $10 \%$. In Sect. 8 we also showed that, in the more dissipative case with $\alpha_{\mathrm{m}}=1$, the accretion flow is disrupted and a highly unsteady outflow is formed if this energy is released inside the disk instead of being radiated.

We also demonstrated how the efficiency of the magnetocentrifugal mechanism, as measured by the ratio of the Poynting flux to the kinetic flux at the disk surface, is affected by the resistive configuration. A higher value of the poloidal and/or toroidal resistivity determines a greater magnetic lever arm, linked to the energy flux ratio as stated by Eq. (53). Our simulations also show that the $\lambda$ parameter, as measured by Eq. (53), is related with a good approximation to the ejection parameter $\xi$ (in the cases where this parameter has been measured):

$\lambda \sim 1+\frac{1}{2 \xi}$

as stated by the self-similar models by Ferreira (1997).

We finally try to test if observations of outflows from classical $\mathrm{T}$ Tauri stars can give some constraint on the parameters of our simulations. The size of the launching region measured in our simulations (from 0.1 to $1 \mathrm{AU}$ ) is consistent with the estimates derived from observations (Bacciotti et al. 2000). On the other hand, the one-sided ejection efficiency inferred from the observations gives values, even with high uncertainty, in the range 0.01-1 (Cabrit 2002), and this result seems to favor our solution characterized by $\left(\alpha_{\mathrm{m}}=1, \chi_{\mathrm{m}}=3\right)$. Even if, in agreement with observations, all our solutions are characterized by a speed at the upper boundary of the computational domain which is a few times the escape speed from the potential well of the central object (Fig. 12), a quantitative comparison with the poloidal speed (Bacciotti et al. 2000) and rotation signatures (Bacciotti et al. 2002; Coffey et al. 2004; Woitas et al. 2005) of the observed outflows is much more difficult, because the size of our computational domain, which reaches a physical scale along $z$ equal to $12 \mathrm{AU}$, is around three times smaller than the distance from the source investigated by current HST observations (>30 AU). As pointed out by Ferreira et al. (2006), a solution characterized by a magnetic lever arm $\lambda \sim 10$ successfully reproduces the values of both the poloidal and toroidal speeds at the currently observed spatial scale: the highest lever arm value found in our simulations $(\lambda \sim 9)$ is observed in the outer part of the launching region of the case $\left(\alpha_{\mathrm{m}}=1, \chi_{\mathrm{m}}=3\right)$ (Fig. 17).

Two specific conclusions and difficulties of our calculations must be mentioned. First, the magnetocentrifugal process appears to operate only with relatively strong magnetic fields in the disk; a strong field tends to inhibit the development of turbulence that could be the origin of magnetic resistivity. Instabilities (shear, Kelvin-Helmholtz) other than magneto-rotational might produce the required turbulence. On the other hand, the resistivity inside a protostellar disk can be extremely high between 0.1 and $10 \mathrm{AU}$, where the grains become the dominant charge carriers (Wardle 1997). The coupling between the accreting plasma and the magnetic field is therefore strongly reduced, perhaps too much so. The second point is that outflow rates measured in our simulations turn out to be rather high, which applies well to jets in star formation regions, where extended bipolar structures are observed. For AGN nuclei one must go to the relativistic limit (which we have not done here), and also discuss how the (possibly subsonic) wind from the disk outer part interacts with the surrounding galaxy.

Acknowledgements. We thank Timur Linde for help in upgrading the MHD version of the FLASH code. C.Z. would also thank Jonathan Ferreira, Zakaria Meliani, and Christophe Sauty for all the valuable suggestions and discussions. The simulations were performed at IDRIS (Orsay) under the Project HPC-EUROPA (RII3-CT-2003-506079), with the support of the European Community - Research Infrastructure Action under the FP6 "Structuring the European Research Area" Program, and partly at CINECA (Bologna, Italy), thanks to the support by INAF. The authors acknowledge support through the Marie Curie Research Training Network JETSET (Jet Simulations, Experiments and Theory) under contract MRTN-CT-2004-005592. This work has been supported in part by the US Department of Energy under grant No. B523820 to the Center of Astrophysical Thermonuclear Flashes at the University of Chicago and by the Italian Ministero dell'Università e della Ricerca under a contract PRIN 2005 to the University of Torino. The software used in this work was in part developed by the DOE-supported ASC/Alliance Center for Astrophysical Thermonuclear Flashes at the University of Chicago.

\section{References}

Anderson, J. M., Li, Z. Y., Krasnopolsky, R., \& Blandford, R. D. 2005, ApJ, 630, 945

Bacciotti, F., Mundt, R., Ray, T. P., et al. 2000, ApJ, 537, 49

Bacciotti, F., Ray, T. P., Mundt, R., Eislöffel, J., \& Solf, J. 2002, ApJ, 576, 222

Balbus, S. A., \& Hawley, J. F. 1998, Rev. Mod. Phys., 70, 1

Blandford, R. D. 1976, MNRAS, 176, 465

Blandford, R. D., \& Payne, D. G. 1982, MNRAS, 199, 883

Cabrit, S. 2002, in Star Formation and the Physics of Young Stars, ed. J. Bouvier, \& J.-P. Zahn, EAS Publ. Ser., 3, 147

Casse, F., \& Ferreira, J. 2000a, A\&A, 353, 1115

Casse, F., \& Ferreira, J. 2000b, A\&A, 361, 1178

Casse, F., \& Keppens, R. 2002, ApJ, 581, 988

Casse, F., \& Keppens, R. 2004, ApJ, 601, 90

Coffey, D., Bacciotti, F., Woitas, J., Ray, T. P., \& Eislöffel, J. 2004, ApJ, 604, 758

De Young, D. S. 2006, ApJ, 648, 200

Dedner, A., Kemm, F., Kroner, D., et al. 2002, JCP, 175, 645

Fendt, C. 2006, ApJ, 651, 272 
Ferrari, A. 1998, ARA\&A, 36, 539

Ferrari, A. 2004, Ap\&SS, 293, 15

Ferreira, J. 1997, A\&A, 319, 340

Ferreira, J., \& Pelletier, G. 1995, A\&A, 295, 807

Ferreira, J., Dougados, C., \& Cabrit, S. 2006, A\&A, 453, 785

Frank, J., King, A. R., \& Raine, D. J. 2002, Accretion Power in Astrophysics (Cambridge: Cambridge University Press)

Fryxell, B., Olson, K., Ricker, P., et al. 2000, ApJS, 131, 273

Giovannini, G. 2004, Ap\&SS, 293, 1

Kato, S. X., Kudoh, T., \& Shibata, K. 2002, ApJ, 565, 1035

Königl, A. 1989, ApJ, 342, 208

Krasnopolsky, R., Li, Z. Y., \& Blandford, R. 1999, ApJ, 526, 631

Krasnopolsky, R., Li, Z. Y., \& Blandford, R. 2003, ApJ, 595, 631

Kuwabara, T., Shibata, K., Kudoh, T., \& Matsumoto, R. 2005, ApJ, 621, 921

Li, Z. Y. 1996, ApJ, 465, 855

Li, H., Lovelace, R. V. E., Finn, J. M., \& Colgate, S. A. 2001, ApJ, 561, 915

Livio, M. 1999, Phys. Rep., 311, 255

Lovelace, R. V. E. 1976, Nature, 262, 649

Lynden-Bell, D. 2003, MNRAS, 341, 1360

Marder, B. 1987, JCP, 68, 48

Meliani, Z., Casse, F., \& Sauty, C. 2006, A\&A, 460, 1

Ogilvie, G. I., \& Livio, M. 2001, ApJ, 553, 158

Ouyed, R., \& Pudritz, R. E. 1997, ApJ, 482, 712
Powell, K. G., Roe, P. L., Linde, T. J., Gombosi, T. I., \& DeZeew, D. L. 1999 , JCP, 154, 284

Pudritz, R. E., Ouyed, R., Fendt, C. \& Brandenburg, A. 2006, in Protostars and Planets V, ed. B. Reipurth, D. Jewitt, \& K. Keil (Tucson: University Arizona Press), 277

Pudritz, R. E., Rogers, C. S., \& Ouyed, R. 2006, MNRAS365, 1131

Reipurth, B., \& Bally, J. 1996, ARA\&A, 39, 403

Sauty, C., \& Tsinganos, K. 1994, A\&A, 287, 893

Sauty, C., Trussoni, E., \& Tsinganos, K. 2002, A\&A, 348, 327

Sauty, C., Trussoni, E., \& Tsinganos, K. 2004, A\&A, 421, 797

Shakura, N. I., \& Sunyaev, R. A. 1973, A\&A, 24, 337

Shu, F., Najita, J., Ostriker, E., et al. 1994, ApJ, 429, 781

Uchida, Y., \& Shibata, K. 1985, PASJ, 37, 515

Ustyugova, G. V., Koldoba, A. V., Romanova, M. M., Chechetkin, V. M., \& Lovelace, R. V. E. 1999, ApJ, 516, 221

Wardle, M. 1997, in Accretion Phenomena and Related Outflows, ed. D. Wickramasinghe, L. Ferrario, \& G. Bicknell (San Francisco: ASP), Proc. IAU Coll., 163, 561

Wardle, M., \& Königl, A. 1993, ApJ, 410, 218

Weber, E. J., \& Davis, L. 1967, ApJ, 148, 217

Woitas, J., Bacciotti, F., Ray, T. P., et al. 2005, A\&A, 432, 149

Zanni, C., Ferrari, A., Massaglia, S., Bodo, G., \& Rossi, P. 2004, Ap\&SS, 293, 99 